Deutschland und das antike Zypern. Beiträge zur Geschichte einer archäologischen Disziplin

Matthias Recke

Citer ce document / Cite this document :

Recke Matthias. Deutschland und das antike Zypern. Beiträge zur Geschichte einer archäologischen Disziplin. In: Cahiers du Centre d'Etudes Chypriotes. Volume 42, 2012. pp. 87-116;

http://www.persee.fr/doc/cchyp_0761-8271_2012_num_42_1_1015

Document généré le 04/05/2017 


\section{DEUTSCHLAND UND DAS ANTIKE ZYPERN Beiträge zur Geschichte einer archäologischen Disziplin}

\section{Matthias RECKE}

Der vorliegende Beitrag versucht, einen Überblick über die aktuelle ZypernArchäologie in Deutschland zu geben. Neben einer Zusammenfassung der Ausgrabungen und Feldforschungen, insbesondere seit dem 2. Weltkrieg, wird der Schwerpunkt auf den deutschen Sammlungen und Museen liegen, die zyprische Antiken beherbergen. Sie können an dieser Stelle nicht im Detail vorgestellt werden, doch soll durch die wichtigsten Eckdaten ihrer Entstehung und Zusammensetzung nicht nur ein Bild der Kypriaka in öffentlichem Besitz, sondern auch ein Baustein zu einer Geschichte des Sammelns zyprischer Altertümer in Deutschland geliefert werden'1. Eine Zusammenstellung von zypern-relevanten Ausstellungen sowie von universitären Promotions- und Forschungsprojekten rundet die Übersicht ab.

\section{AUSGRABUNGEN UND FELDFORSCHUNGEN}

Die frühen Grabungstätigkeiten auf Zypern, insbesondere seit dem Beginn der britischen Herrschaft 1878, sind in letzter Zeit verschiedentlich zusammengestellt worden und sollen hier nicht ausführlich behandelt werden. Max Ohnefalsch-Richter, der eine zentrale Rolle für die zyprische Archäologie des ausgehenden 19. Jahrhunderts spielt, war als Privatmann für verschiedene Geldgeber tätig, deren Ziel vorrangig im Finden von musealen oder auf dem Markt veräußerbarer Kunstgegenstände lag. OhnefalschRichter entwickelte, wie zuletzt H. Matthäus und S. Rogge herausgestellt haben², im Laufe seiner Tätigkeit jedoch zunehmend wissenschaftliche Ambitionen und verfolgte durch seine rege Publikationstätigkeit, seine Promotion und ein (nicht abgeschlossenes)

1. Eine entsprechende Übersicht wäre ohne die bereitwillige Unterstützung der Kollegen an Museen und Universitäten nicht möglich gewesen. Angesichts der rund 70 Institutionen in Deutschland, die zyprische Antiken beherbergen, soll auf eine namentliche Danksagung verzichtet werden.

2. Matthäus 2009; Rogge 2007a. 
Habilitationsprojekt ${ }^{3}$ neben den merkantilen Interessen auch archäologische Fragestellungen und strebte die wissenschaftliche Anerkennung der Fachwelt an. Wichtig für seine Selbstwahrnehmung war dabei die Zusammenarbeit mit verschiedenen deutschen Gelehrten, die durch ihre Position und bisherige wissenschaftlichen Leistungen ein gewisses Ansehen genossen, oder als Hoffnungsträger galten. So finden wir Grabungen und Unternehmungen unter seiner Leitung oder zumindest maßgeblicher Mitwirkung, die stärker wissenschaftlichen Charakter hatten, ab 1885 (zusammen mit Ferdinand Dümmler) und vor allem für die Berliner Museen 1889 (zusammen mit Adolf Furtwängler). 1890 bereist Wilhelm Dörpfeld zusammen mit Ohnefalsch-Richter die Insel und erstellt eine umfassende Photodokumentation für das Deutsche Archäologische Institut in Athen ${ }^{4}$.

Eine seit 1907 von ihm geplante Grabung in Rantidi in der Umgebung von Paphos kam nicht zustande, stattdessen grub Robert Zahn im September und Oktober 1910 hier für die Berliner Akademie 5 .

\section{Ausgrabungen nach dem 2. Weltkrieg}

Es ist bezeichnend, wie stark Max Ohnefalsch-Richter die deutschen Forschungen auf Zypern geprägt hat. Ohne ihn als Initiator und Kontaktmann vor Ort sollte es bis weit nach dem 2. Weltkrieg dauern, dass deutsche Archäologen wieder auf Zypern Ausgrabungen unternahmen.

Alt-Paphos. Die bereits 1888 durch den Cyprus Exploration Fund begonnenen und zwischen 1950-1955 durch die Universität St. Andrews und das Museum Liverpool fortgeführten Ausgrabungen in Kouklia / Palaepaphos wurden 1966 durch Franz Georg Maier von der Universität Konstanz mit Unterstützung des Deutschen Archäologischen Instituts wieder aufgenommen. Maier setzte die Ausgrabungen auch nach seiner Berufung 1973 an die Universität Zürich fort und arbeitete bis 1995 vor Ort. Ziel der Ausgrabungen war die Erforschung der Stadtgeschichte anhand sorgfältiger Beobachtungen zur Topographie, Siedlungsstruktur und urbanistischen Entwicklung. Neben dem in die Bronzezeit zurückreichenden Aphrodite-Heiligtum waren das Nordosttor und die persische Belagerungsrampe ein besonderer Schwerpunkt, doch wurde durch eine enge Verknüpfung von Survey und Flächengrabung auch die frühbyzantinische und mittelalterliche Geschichte des Ortes erforscht.

Die Ergebnisse der Feldforschungen und der Materialbearbeitung werden in der Reihe „Ausgrabungen in Alt-Paphos“ vorgelegt, die inzwischen bei Band 8 angelangt

3. Brönner 1999, p. 110.

4. Die 105 verfügbaren Motive sind im Archäologischen Anzeiger 1891, p. 89-91, aufgelistet und umfassen neben rein archäologischen Aufnahmen von Ausgrabungen und Funden auch volks- und landeskundlich Bilder; sie sind heute von bedeutendem historischem Wert.

5. Vgl. Maier 1983, p. 2. 
ist $^{6}$. Mehrere Bände, insbesondere die Publikation der Skulpturenfunde aus der Belagerungsrampe, stehen noch aus.

Tamassos. Ebenfalls mit Unterstützung und im Auftrag des Deutschen Archäologischen Instituts begann 1970 Hans-Günter Buchholz von der Universität Gießen mit Feldforschungen in Tamassos. Erklärtes Ziel der bis 1980 jährlich stattfindenden Kampagnen war es, das von Max Ohnefalsch-Richter hinterlassene Manuskript "Tamassos und Idalion" durch gezielte Nachgrabungen zur Publikation vorzubereiten und eine Auswertung der Funde von dessen verschiedenen Grabungen 1885, 1889 und 1894 vorzunehmen. Obwohl die Grabungen in vieler Hinsicht Kontrollcharakter hatten ${ }^{7}$, wurden die Aktivitäten auch auf das weitere Umland ausgedehnt. In Aufsatzform hat Buchholz eine Vielzahl von Aspekten in einer Fülle von Einzelbeiträgen behandelt ${ }^{8}$. Nachdem 1996 eine erste monographische Synthese vorgelegt worden war ${ }^{9}$, erfolgte 2010 die Publikation des unter internationaler Beteiligung verfassten ersten Bandes der endgültigen Grabungspublikation ${ }^{10}$. Darin werden insbesondere die Nekropolen von Tamassos behandelt. Weitere Bände sind kurz vor der Fertigstellung oder zumindest weit fortgeschritten ${ }^{11}$; ihre Herausgabe erfolgt seit dem Tod von Hans-Günter Buchholz 2011 durch H. Matthäus (Erlangen) und M. Recke (Gießen).

Nea Paphos. 1987 erfolgte unter der Leitung von Günter Grimm eine Ausgrabung der Universität Trier am Theater von Nea Paphos, die in Kooperation mit der polnischen Mission unter Wiktor A. Daszewski stattfand. Die Arbeiten wurden nach der ersten Kampagne allerdings nicht fortgesetzt und sind bis auf eine kurze Zusammenfassung durch V. Karageorghis unpubliziert ${ }^{12}$.

Hala Sultan Tekke. Seit 2011 gräbt die Universität Gießen unter Leitung von Matthias Recke in Hala Sultan Tekke, in Kooperation mit der Universität Brüssel (VUB) unter Karin Nys. Die bisherigen Arbeiten dienen der Endpublikation eines Gebäudekomplexes, der bereits 1980 durch Paul Åström von der Universität Göteborg entdeckt und zum Teil großflächig freigelegt worden war, doch soll die Kooperation darüber hinaus fortgesetzt werden. Ziel ist neben der Klärung der Bau- und Architekturgeschichte dieses Areals ein besseres Verständnis des urbanistischen Systems spätbronzezeitlicher Städte auf Zypern ${ }^{13}$.

6. Näf 2013. Die Reihe umfasst auch die Publikation einer Grabung des Departments: Karageorghis 1983.

7. Buchholz 1989, p. 21-22.

8. Vgl. seine Bibliographie und den Nachtrag in Matthäus 2011, p. 15-16.

9. Buchholz, Untied 1996.

10. Buchholz 2010.

11. H. Matthäus zur Königsnekropole; M. Recke zu den außerstädtischen Heiligtümern. Die Bearbeitung der Stadtgrabung wird aus den von Buchholz hinterlassenen Vorarbeiten erfolgen.

12. Karageorghis 1988, p. 835-836.

13. Nys, Recke 2011, p. 72-83. 


\section{ZUR GESCHICHTE DES SAMMELNS ZYPRISCHER ANTIKEN IN DEUTSCHLAND}

Wie die im Anhang aufgeführte Auflistung zeigt, gibt es in Deutschland eine Fülle von öffentlichen Sammlungen, die zyprische Antiken besitzen. Von wenigen Ausnahmen abgesehen sind die Bestände in der Regel aber nur unzureichend oder gar nicht publiziert und in den Depots verwahrt.

Mit Ausnahme der Berliner Antikensammlung, die auch eigene Grabungen auf der Insel unternommen hat, sind die meisten Sammlungen größtenteils im ausgehenden 19. Jahrhundert durch Ankäufe aus dem Kunsthandel entstanden. Ein Überblick über ihre Entstehungsgeschichte, sofern diese dokumentiert ist oder erschlossen werden kann, zeigt, dass die Geschichte des Sammelns zyprischer Antiken in mehrere charakteristische Phasen untergliedert werden kann.

Unmittelbar mit dem Herauskristallisieren einer Zypern-Archäologie im 19. Jh. und ausschlaggebend für eine zunehmend wissenschaftliche Beschäftigung mit der materiellen Kultur ist das Sammeln entsprechender Ausgrabungsgegenstände verbunden. Anders als die Erforschung griechischer und römischer Altertümer hat das Sammeln zyprischer Antiken keine jahrhundertealte, im Humanismus der Renaissance gründende Tradition. Erste Kypriaka in deutschen Sammlungen lassen sich erst um die Mitte des 19. Jhs. fassen und sind dann in der Regel mit Namen von gelehrten Orientreisenden zu verbinden. So hat der Altertumswissenschaftler Ludwig Ross (1806-1859) während seiner Reisen 1845 auf Zypern nicht nur Antiken für die Berliner Museen erworben und den Ankauf der berühmten Sargon-Stele aus Kition vermittelt, sondern auch kleinere Objekte für sich selbst gesammelt ${ }^{14}$. Seine Sammeltätigkeit ist charakteristisch für die Mitte des 19. Jhs.: Zumeist handelt es sich um Gelehrte, die während ihrer Forschungsreisen bei Gelegenheit auch Antiken erwarben - kleinere für sich selbst, größere für die heimischen Museen. Der systematische Erwerb von Antiken war aber nicht der Hauptzweck solcher Unternehmungen, und sie belieferten auch nicht den freien Kunstmarkt.

Julius August Schönborn (1801-1857), der Entdecker des Heroons von Trysa in Lykien, hat auf seiner Zypernreise 1851 rund 20 Vasen und Terrakotten sowie die Bronzestatuette eines Satyrn erworben und sie nach seiner Rückkehr dem Berliner

14. Zur Zypern-Reise Ross vgl. Masson, Hermary 1988, p. 3-10; Mehl 2009, p. 153-187. Obwohl Ross auf Zypern selbst nicht gegraben hat, sind ihm dort einige bedeutsame Entdeckungen gelungen, etwa die Lokalisierung des antiken Tamassos. Sein für diese Zeit noch recht ungewöhnliches Interesse an den Kulturen des östlichen Mittelmeerraumes wird bereits in seinem 1841 in Athen erschienenem Handbuch der Kunstarchäologie deutlich, das die orientalischen Zivilisationen Ägyptens, der Levante und Kleinasiens als Vorgänger und Grundlage der griechischen Kunst verstand, vgl. Fittschen 2005, p. 215-261. - Eine der in Idalion erworbenen Terrakotta-Statuetten bildete Ross 1852 in der Publikation seines Reiseberichts ab, vgl. Mehl 2009, p. 175 Abb. 4. Ein weibliches Kalksteinköpfchen, ebenfalls aus Idalion, stiftete er 1851 in die Sammlung des Archäologischen Instituts der Universität Halle (Inv. 100), wo er seit 1845 bis zu seinem Tod 1859 lehrte. Aus seinem Nachlass gelangten 1860 fünf zyprische Bronzen in die Berliner Antikensammlung, darunter eine kleine hellenistische Bronzestatuette aus Tamassos, vgl. Franken 2011, p. 54. 
Museum übereignet ${ }^{15}$. Als einer der letzten Vertreter dieser Kategorie ist der Archäologe Ferdinand Dümmler (1859-1896) anzusehen, der 1885 die Insel besuchte. Anders als die bisher angesprochenen Gelehrten hat Dümmler selbst auch einige kleinere Ausgrabungen unternommen, bei denen er von Max Ohnefalsch-Richter unterstützt wurde. Obwohl ihm im Zuge der Fundteilung ein Teil der Funde zugestanden hätte, verzichtete er explizit auf seinen Anteil, um Grabzusammenhänge nicht zu trennen und für künftige Forschungen zu erhalten ${ }^{16}$. Seine im örtlichen Kunsthandel erworbenen Antiken gelangten 1896 als Schenkung an das Berliner Museum für Vor- und Frühgeschichte und, aus seinem Nachlass, 1899 an das Museum in Kassel.

Die Möglichkeit, in Mitteleuropa zyprische Antiken zu erwerben, ohne sich den Strapazen einer Reise nach Zypern selbst zu unterziehen, bot sich im größeren Maßstab erst ab 1870, als Luigi Palma di Cesnola seine umfangreiche Sammlung auflöste und verschiedenen Museen und Kunsthändlern zum Verkauf anbot. Cesnola, der seit 1865 als amerikanischer Konsul auf Zypern tätig war, hatte über Jahre hinweg ohne wissenschaftlichen Anspruch systematische Raubgrabungen auf der ganzen Insel durchgeführt, die den Zweck hatten, sammel- und verkaufswürdige Objekte zu entdecken ${ }^{17}$.Ab 1870 ließ er umfangreiche Bestände seiner insgesamt rund 35.000 Objekte umfassenden Sammlung auf mehreren Auktionen in Paris und London versteigern, verkaufte aber auch direkt an Museen und Sammlungen. Auf diese Weise gelangten zyprische Objekte in die Münchener Antikensammlung und in Privatsammlungen wie die des Frankfurter Kaufmanns Carl Anton Milani (1820-1882), der mehrere Kalksteinköpfe und Terrakotten erwarb, die heute zum Grundstock der Frankfurter Zypernsammlung gehören. Cesnola handelte aber - wie auch sein jüngerer Bruder Alessandro - auch vor Ort mit Antiken, insbesondere in der bedeutendsten zyprischen Hafenstadt dieser Zeit, Larnaka. 1869 verkaufte er dort über Carl Friederichs den Berliner Museen 180 Objekte ${ }^{18}$. Larnaka war der Sitz der Diplomaten, Bankiers und Kaufleute und galt als Zentrum des (von eben diesen Gruppen durchgeführten) Antikenhandels, was dazu führte, dass die Umgebung der Stadt systematisch durch Raubgrabungen geplündert wurde ${ }^{19}$.

15. Brehme 2002, p. 11.

16. Die entsprechenden Komplexe wurden allerdings durch die verschiedenen Reorganisationen und Umzüge des Cyprus-Museums schon bald darauf auseinander gerissen, so dass sie bereits in dem 1899 erschienenen Katalog des Cyprus Museums von Myres und Ohnefalsch-Richter als nicht-identifizierbar galten: Myres, Ohnefalsch-Richter 1899, p. VI-VII.

17. Zum Verkauf der beiden Cesnola-Sammlungen vgl. Masson 1996,p. 3-27; zu ihrer Geschichte Karageorghis 2000, p. 3-15.

18. Masson 1996, p. 4; Brehme 2002, p. 11.

19. Bereits Ludwig Ross hatte hier in Larnaka 1845 vom österreichischen Konsul eine Anzahl von Terrakotten zum Geschenk erhalten, vgl. Mehl 2009, p. 174. Generell vgl. Masson 1992, p. 123-154. - Auch der durch seine Sammlung bis heute bekannte Bankier Dimitris Pierides, Vizekonsul von Großbritannien, war als Vermittler von Antiken in Larnaka tätig; außerdem war er ausländischen Forschern, die die Insel bereisten, behilflich, vgl. Buchholz 1989, p. 9. 
Der enorme Aufschwung, den das Sammeln zyprischer Antiken in Deutschland ab den 80er Jahren des 19. Jhs. erlebte, wurde durch zwei Faktoren unterstützt. Zum einen sorgte die Gesetzeslage auf Zypern dafür, dass große Mengen von Ausgrabungsgegenständen in den Handel gelangten. Durch verschiedene Gesetzgebungen hatte die osmanische Inselverwaltung bereits seit 1869 versucht, die ungebremsten Ausgrabungstätigkeiten durch Vergabe von Lizenzen zu regulieren und zu kontrollieren. Die 1874 eingeführte Regel der Fundteilung (bei der ein Drittel der Funde dem Ausgräber, ein Drittel dem Landbesitzer und eines der Regierung zustand) führte dazu, dass große Mengen von Antiken ganz legal dem Kunsthandel zuflossen. Ein Exportverbot von Antiken aus dem Jahre 1884 wurde von der britischen Verwaltung, der die Insel seit 1878 unterstand, nicht anerkannt, so dass de facto weiterhin die Regelung der Fundteilung praktiziert wurde ${ }^{20}$.

Der zweite Faktor, der die gewaltige Fluktuation zyprischer Antiken in deutschen Sammlungen des ausgehenden 19. Jhs. erklären kann, liegt in der Person Max OhnefalschRichters (1850-1917) und seiner vielfältigen Aktivitäten begründet. Nachdem er seit 1878 zunächst als Berichterstatter für mehrere Zeitungen auf Zypern tätig gewesen war, agierte er seit 1879 als Ausgräber für verschiedene Institutionen und Privatpersonen. Permanent in Geldnot, hat er zumeist nicht auf eigene Kosten gegraben, es aber in der Regel verstanden, die weiterhin praktizierte Fundteilung zu seinen Gunsten anzuwenden. Seine Ausgrabungen, seine vielfältigen Veröffentlichungen und seine Tätigkeit als Kunstagent haben bereits von den Zeitgenossen stark voneinander abweichende Beurteilungen erfahren, und bis heute ist die Einschätzung seiner Person kontrovers ${ }^{21}$. Da die Masse zyprischer Antiken, die sich in deutschen Sammlungen befinden, entweder aus seinen Grabungen stammt oder von ihm vermittelt wurde, sind seine Spuren quasi überall zu finden.

\section{Ohnefalsch-Richter und die Entstehung der großen Museumssammlungen}

Insgesamt lassen sich vier Kategorien unterscheiden, nach denen Antiken durch Ohnefalsch-Richter in deutsche Sammlungen gelangten: a) durch direkten Verkauf von Objekten aus den von ihm durchgeführten Grabungen, die ihm (oder dem Finanzier der Grabung) im Rahmen der Fundteilung zustanden; b) durch Vermittlung von Objekten aus Grabungen anderer oder von Gegenständen, die er selbst ohne weitere Angaben auf Zypern aufgekauft hatte; c) durch Belieferung von Kunsthändlern und Auktionen als Zwischenverkäufer; d) durch direkte Beteiligung deutscher Institutionen im Rahmen der Beauftragung und Finanzierung der Ohnefalsch-Richter'schen Ausgrabungen. Die entsprechenden Museen erwarben dadurch gemäß der praktizierten Rechtsauslegung das Anrecht auf ein Drittel der zu erwartenden Funde.

Die Berliner Antikensammlung, für die sich alle diese vier Kategorien nachweisen lassen, verdankt Ohnefalsch-Richter einen gewaltigen Zuwachs ihrer Bestände zyprischer

20. Zur Gesetzgebung ausführlich Zachariou-Kaila 2009, p. 271-293.

21. Zuletzt Matthäus 2009, p. 115-151; Buchholz 1989, p. 3-27; Buchholz 2000, p. 91-101; Brönner 2004/05, p. 320-332; Fivel 1996, p. 29-35. 
Antiken: So stammen rund 500 Objekte, die er 1887 an die Berliner Museen verkaufte, aus seiner für Charles Watkins von der Imperial Ottoman Bank in Larnaka durchgeführten Grabung im Aphrodite-Heiligtum von Idalion ${ }^{22}$. Ein weitaus größerer Teil der Berliner Bestände stammt jedoch aus Grabungen, die von Seiten des Museums finanziert worden waren. Auf diesem Weg gelangten 300 Objekte aus der Idalion-Grabung 1894 und rund 800 Objekte aus den Grabungen 1889 in Tamassos, bei denen er zeitweise durch Adolf Furtwängler unterstützt wurde, nach Berlin ${ }^{23}$. Weitere 300 zyprische Objekte erwarb das Berliner Museum 1887 auf einer Pariser Kunstauktion ${ }^{24}$; sie stammen aus der Nekropole von Marion, die Ohnefalsch-Richter in einer mit C. Christian gegründeten „Ausgrabungsgesellschaft“ 1886 für Charles Watkins, Direktor der Imperial Ottoman Bank in Larnaka untersucht hatte. Und schließlich vermittelte Ohnefalsch-Richter auch Funde nach Berlin, die er nicht selbst ausgegraben hatte, so etwa rund 170 Objekte aus einem Heiligtum bei Limniti. Insgesamt sind so binnen weniger Jahre weit über 2000 zyprische Objekte in die Berliner Antikensammlung gelangt ${ }^{25}$.

Dieses Muster lässt sich, in geringerem Umfang, auch an anderen Orten feststellen. So grub Ohnefalsch-Richter mit Mitteln der Virchow-Stiftung 1894 nochmals in der Lambertis-Nekropole von Tamassos; die Funde dieser Grabung befinden sich heute im Akademischen Kunstmuseum in Bonn und in der Berliner Antikensammlung (sowie in den Sammlungen des Cyprus Museums Nikosia und in Cambridge). Fundstücke aus der Mnason-Nekropole von Tamassos, ebenfalls 1885 und 1889 ergraben, befinden sich, außer in Nikosia, auch in Dresden und im Römisch-Germanischen Zentralmuseum in Mainz $^{26}$.

Bei der Mehrzahl der durch Ohnefalsch-Richter arrangierten Verkäufe sind allerdings die genauen Fundumstände der vermittelten Objekte unbekannt, und selbst die Fundorte nicht immer überliefert. Dies ist zum Teil sicher durch die wenig sorgfältige Dokumentation während der Grabung begründet, zum andern aber auch durch den Umstand bedingt, dass es sich in der Regel um nachträglich zusammengestellte Konvolute verschiedener Fundorte handelt. Der Kauf von gemischten Objektgruppen war für Museen und Sammlungen insofern attraktiv, als sie dadurch einen diachronen Querschnitt der materiellen Kultur Zyperns von der Bronzezeit bis in hellenistisch-römische Zeit erhielten, bei dem die wichtigsten Materialgattungen exemplarisch vertreten waren ${ }^{27}$.

22. Brehme 2002, p. 12. Über 150 Objekte der Idalion-Grabung von 1885 gelangten 1887 durch Vermittlung Adolf Furtwänglers an das Museum von Karlsruhe, darunter zahlreiche Kalksteinstatuetten und großformatige Terrakotten, vgl. Schürmann 1984, p. 4-5.

23. Brehme 2002, p. 12. Zu den verschiedenen Tamassos-Grabungen Ohnefalsch-Richters jetzt Buchholz 2010.

\section{Fröhner 1887.}

25. Brehme 2002, p. 11-12.

26. Buchholz 2011, p. 91.

27. Brönner 2001, p. 201-205. 
Auf diese Weise wurde der Grundstock zu weiteren Zypernbeständen großer deutscher Museen gelegt: Rund 200 Objekte des Badischen Landesmuseums gelangten in drei Etappen zwischen 1887 und 1895 nach Karlsruhe. Teile der Ankäufe stammen nachweislich von verschiedenen Grabungen Ohnefalsch-Richters (Idalion, Tamassos, Ledra ${ }^{28} .1892$ wurde eine entsprechende Sammlung nach Dresden verkauft ${ }^{29}$, und auch die Antikensammlung Mannheim erwarb zwischen 1895 und 1897 eine entsprechende Kollektion Ohnefalsch-Richters. Das Römisch-Germanische Zentralmuseum in Mainz erhielt 1898 seitens der Virchow-Stiftung Berlin eine Sammlung von über 240 Antiken aus Zypern geschenkt, die ebenfalls von Ohnefalsch-Richters Ausgrabungen stammen ${ }^{30}$.

\section{Privatsammlungen des ausgehenden 19. Jahrhunderts}

Wie Cesnola hat Ohnefalsch-Richter nicht nur an Museen verkauft, sondern auch an Privatpersonen. Hier lässt sich, anders als bei den musealen Auswahlkollektionen, ein stärkeres Eingehen auf die Wünsche der jeweiligen Kunden erkennen. So hat er dem Münchner Maler Julius Naue (1835-1907), der zeitweilig für ihn gezeichnet hat, neben Keramik insbesondere Bronzenwaffen besorgt. Ein frühbronzezeitlicher Grabfund aus Psematismenos bei Larnaka mit mehreren Dolchen und einem langen Griffangelschwert ist von Naue, der sich als Privatgelehrter der Erforschung der Bayerischen Vorgeschichte widmete, in seinem maßgeblichen Buch "Die vorrömischen Schwerter aus Kupfer, Bronze und Eisen" (München 1903) publiziert worden.

Der Straßburger Archäologe Robert Forrer (1866-1947) bezog über OhnefalschRichter neben Gefäßen und Terrakotten ebenfalls vor allem Metallobjekte (Waffen, Werkzeuge und Schmuck). Forrer hat diese Stücke in verschiedenen Publikationen, unter anderem in seinem umfangreichen "Reallexikon der prähistorischen, klassischen und frühchristlichen Altertümer" von 1907 und in seiner "Urgeschichte des Europäers" von 1908 zur Illustration verwendet.

Heinrich Frauberger, der Direktor des Düsseldorfer Gewerbemuseums, erwarb gezielt Goldschmuck und konnte während einer Orientreise über 200 Objekte durch Vermittlung Ohnefalsch-Richters auf Zypern erwerben; er hat sie als Anhang zu dessen monumentalem Werk "Kypros, die Bibel und Homer" 1893 in Auswahl publiziert.

Von großer Bedeutung war Ohnefalsch-Richters Anwesenheit als Verkäufer auf der Berliner Gewerbeausstellung von 1896. Hier hat er nach eigener Aussage "neben vielen interessanten Museumsstücken auch noch Tausende zählende kleine billige decorative Stücke" zum Verkauf angeboten ${ }^{31}$. Was alles zwischen dem 1. Mai und dem 15. Oktober 1896 über den Ladentisch ging und in ungenannten Privatsammlungen landete, lässt sich in keiner Weise mehr rekonstruieren. Über zwei Millionen Besucher wurden gezählt,

28. Schürmann 1984, p. 4.

29. Knoll 1996/7, p. 11.

30. Vgl. Behn 1913, p. 67-97.

31. Brönner 1999, p. 110. 
und Ohnefalsch-Richter hatte zeitweise Probleme mit der Nachschublieferung, so dass er sich gezwungen sah, hochwertige Stücke unter Wert zu verkaufen. Es ist anzunehmen, dass vieles, was sich im ausgehenden 19. und frühen 20. Jh. an zyprischen Stücken in deutschen Privatsammlungen befand, ursprünglich hier erstanden wurde. Die nach Ende der Gewerbeausstellung unverkauften Objekte wurden schließlich, durch Vermittlung Rudolf Virchows, von Valentin Weisbach (1843-1899) erworben ${ }^{32}$. Nach diesem Zeitpunkt sind keine weiteren Verkäufe Ohnefalsch-Richters - weder an Museen noch an Privatpersonen - mehr belegt.

\section{Von der Privatsammlung ins Museum}

Es ist eine bekannte Tatsache, dass Privatsammlungen in der Regel über kurz oder lang in öffentlichen Besitz übergehen, und so sind die hier beispielhaft erwähnten Sammlungen längst Teil großer Museumsbestände: Die 1896 erworbene Sammlung von Valentin Weisbach wurde von diesem bereits zwei Jahre später (und auf Betreiben Ohnefalsch-Richters) 1898 dem Völkerkundemuseum in Leipzig gestiftet. Die Sammlung von Julius Naue, die neben rund 120 zyprischen Objekten auch umfangreiche Antiken aus Ägypten, Griechenland und Italien umfasste, wurde nach dessen Tod in München versteigert ${ }^{33}$. Zyprische Antiken der Sammlung Naue befinden sich heute etwa in den Antikensammlungen der Universitäten Jena und Erlangen sowie im Badischen Landesmuseum Karlsruhe ${ }^{34}$.

Auch der Archäologe Robert Forrer hat, als er sich anderen wissenschaftlichen Fragestellungen zuwandte, die zyprischen Bestände seiner Sammlungen verkauft; sie befinden sich heute u.a. im Martin-von-Wagner-Museum der Universität Würzburg, als Grundstock der 1913 gegründeten archäologischen Sammlung des Ruhrland-Museum (jetzt RuhrMuseum) Essen und im Römisch-Germanischen Zentralmuseum in Mainz.

\section{Der Antikenhandel in Athen, Smyrna und Kairo}

Obwohl Ohnefalsch-Richter zweifellos den bedeutendsten Anteil an der Erwerbung zyprischer Antiken in deutschen Sammlungen hat und, neben Kunsthandlungen wie Helbing in München oder Lederer in Berlin, die entsprechendes Material wiederund weiterverkauften, die Hauptquelle des innerdeutschen Kunsthandels darstellte, gab es auch im Ausland Möglichkeiten, zyprische Ausgrabungsobjekte zu erwerben. Dies gilt natürlich für Zypern selbst, wo weiterhin ein schwunghafter Antikenhandel betrieben wurde. Daneben etablierten sich aber auch Athen, Smyrna und sogar Kairo als

32. Brönner 1999, p.118-119; Brönner 2004/5, p. 320-332.

33. Helbing 1908.

34. Neben zwei mittelbronzezeitlichen Gefäßen, die aus der Nachlassauktion von 1908 erworben wurden, befinden sich dort ein archaisches Fläschchen und eine hellenistische Schalenlampe, die Naue bereits 1885 als Schenkung an das Karlsruher Museum gegeben hatte, vgl. Schürmann 1984, p. 4. Bislang verschollen ist der erwähnte Grabfund aus Psematismenos, doch sind Kopien der Objekte im Römisch-Germanischen Zentralmuseum in Mainz vorhanden, vgl. Behn 1913, p. 93, p. 95. 
Umschlagplatz für zyprische Antiken. So berichtete Otto Rubensohn 1905 aus Ägypten: "Hier in Kairo sind außerordentlich viele cyprische Vasen in den Handel gekommen, die aber nicht in Ägypten gefunden sind, sondern vor kurzem von einem Händler aus Cypern hierher geschafft sind" 35 . Diese Entwicklung steht möglicherweise auch mit dem neuen Antikengesetz auf Zypern in Verbindung, das 1905 erlassen wurde ${ }^{36}$.

Obwohl Stücke aus dem Kunsthandel in Athen (etwa für das Museum vonAachen 1906), Smyrna (Sammlung Vasel, jetzt Museum Braunschweig) oder Kairo (1906 für Dresden) auch für öffentliche Kunstmuseen erworben wurden, fällt auf, dass die Mehrzahl der für diese drei Zentren dokumentierten Ankäufe für universitäre Lehrsammlungen getätigt wurde. Zyprische Antiken aus dem Athener Kunsthandel finden sich beispielsweise in den Universitätssammlungen von Bonn (1902), Erlangen (1903) und Kiel (1906). Siegfrid Loeschke erwarb im Auftrag seines Vaters Georg für das Berliner WinckelmannInstitut zyprische Stücke in Athen (1912/13) und in Smyrna (1911 und 1912). Stücke aus dem Handel in Kairo gelangten nach Bonn (1902), Hildesheim (durch Wilhelm Pelizaeus) und aus der Sammlung Herold in die Universitätssammlung von Rostock. Der Archäologe Bruno Sauer hat auf seiner Ägyptenreise 1909 zyprische Stücke nicht nur für die Universität Kiel, sondern auch für seine Privatsammlung erworben, die später zum Teil in den Besitz des bedeutenden Zypern-Archäologen Hans-Günter Buchholz (19192011) übergingen und sich jetzt in der Sammlung des Biblisch-Archäologischen Instituts der kirchlichen Hochschule Wuppertal befinden. Die zyprischen Antiken der Sammlung Rubensohn, 1905 in Kairo erworben, befinden sich inzwischen in der Archäologischen Sammlung der Universität Münster.

All diese Erwerbungen umfassten aber in der Regel nicht ausschließlich zyprische Antiken, sondern zumeist Werke der griechisch-römischen Kleinkunst, die mit zufälligen Stilproben der "Randgebiete" ergänzt wurden. Auf diese Weise gelangten im frühen 20. Jh. beispielsweise auch kleinasiatische Keramik der Frühbronzezeit, anatolisch-eisenzeitliche Funde, syro-palästinensisches Material und Ägyptiaka in die Lehrsammlungen der Klassisch-Archäologischen Institute.

Diesen Charakter besitzen auch die Privatsammlungen des frühen 20. Jhs., so weit wir sie heute fassen können. Dies ist in der Regel dann der Fall, wenn diese Sammlungen an öffentliche Museen verkauft oder ihnen gestiftet werden. Meist tauchen die zyprischen Antiken als Schenkungen inter alia auf, und in der Regel handelt es sich dabei um Einzelstücke oder kleinere Konvolute. Die Sammler waren überwiegend wohlhabende Bürger, die Kunst und Kultur gegenüber aufgeschlossen waren. Namen bedeutender Fabrikanten und Industrieller tauchen hier auf, die sich als Mäzene ihrer heimischen Museen hervortaten und ihre Sammlungen oder Teile davon stifteten. In der Regel ist nicht bekannt, wo sie ihre zyprischen Antiken erworben haben. Meist dürfte dies im

35. Rubensohn 1905, p. 69.

36. Zachariou-Kaila 2009, p. 291. 
Berliner, Kölner oder Münchner Kunsthandel erfolgt sein, und höchstwahrscheinlich befinden sich darunter auch zahlreiche Stücke, die durch Ohnefalsch-Richter nach Deutschland gekommen sind. In Leipzig etwa schenkten die Verleger Brockhaus, Credner und Giesecke im Jahre 1907 (sowie Marianne Brockhaus im Jahre 1910) der Universitätssammlung zyprische Antiken. 1910 stiftete der Stuttgarter Unternehmer Ernst von Sieglin mehrere zyprische Vasen und Terrakotten nach Dresden. Mit Mitteln Sieglins erwarb auch die Antikensammlung in Tübingen durch Karl Hähnle 1913 drei zyprische Vasen. 1917 erwarb das Hamburger Museum für Kunst und Gewerbe die Sammlung des Hamburger Kaufmanns Johannes W. F. Reimers mit über 1500 antiken Objekten, darunter auch etliche Kypriaka. Im selben Jahr gelangte die umfangreiche Sammlung des Münchner Malers Gabriel von Max mit zahlreichen Kypriaka in den Besitz des Mannheimer Museums.

Dass der Erwerb von zyprischen Antiken nicht auf das Großbürgertum bedeutender Städte begrenzt ist, zeigt etwa die Sammlung des Fabrikanten Wilhelm Gail in Gießen, die um 1900 entstand ${ }^{37}$ und neben provinzialrömischen und lokalarchäologischen Funden auch mehrere kleinformatige zyprische Gefäße der mittleren Bronzezeit umfasste.

\section{Sammlungen deutscher Diplomaten}

Eine gewisse Sonderstellung unter den deutschen Privatsammlungen nimmt die über 100 zyprische Objekte umfassende Sammlung Paul von Tischendorf ein, die sich bereits seit 1914 als Dauerleihgabe im Kestner-Museum befand und schließlich 1952 vom Museum erworben wurde. Sie ist wahrscheinlich als Diplomatensammlung im Orient entstanden und damit, anders als die meisten Privatsammlungen dieser Zeit, in ihrer Entstehungsgeschichte unmittelbar mit der Biographie ihres Sammlers verknüpft.

Bei anderen Sammlungen deutscher Diplomaten ist die Kenntnis von ihrer Sammlungstätigkeit weitaus geringer. So besaß Wilhelm Fabricius (1882-1964), der 1908 in Gießen als Jurist promoviert worden war, einen großformatigen zyprischen Frauenkopf aus Kalkstein, der aus dem kilikischen Mersin stammen soll ${ }^{38}$. Fabricius war als Mitarbeiter des Auswärtigen Dienstes 1925-1936 in Konstantinopel und Ankara tätig und soll den Kopf dort zwischen 1930 und 1932 erworben haben, doch fehlen genauere Informationen $^{39}$.

Immerhin wird aus den angeführten Beispielen deutlich, dass das Sammeln zyprischer Antiken für Angehörige des diplomatischen Dienstes durchaus üblich war und sich deren Sammlungen nicht auf griechisch-römische Altertümer beschränkten. Neben dem

37. Durch die Vernichtung der entsprechenden Archivunterlagen 1944 lässt sich die Geschichte der Gail'schen Sammlungen, die Teil des Oberhessischen Museums Gießen waren, nur ansatzweise rekonstruieren. Die Kenntnis der zyprischen Antiken dieser Sammlung beruht auf der Inventarbucheintragung einer Schenkung des Gießener Museums an das Römisch-Germanische Zentralmuseum in Mainz im Jahre 1938.

38. Neugebauer 1938, p. 10.

39. Cassimatis 1981, p. 160-168. - Der Kopf war dann 1997 und 2001 bei Christie's in New York, vgl. Lafli, Recke 2005, p. 16 Anm. 45. 
(aktiven) Kauf muss man allerdings auch damit rechnen, dass sie entsprechende Stücke als Geschenke erhielten. Dies ist beispielsweise bei der Sammlung des Diplomaten von Schmoller der Fall, die ihren Ursprung in den 50er und 60er Jahren des 20. Jhs. hat. Sie enthält zahlreiche zyprische Antiken, die Gustav von Schmoller (1907-1991) von Erzbischof Makarios in Anerkennung seiner Dienste als Geschenk erhalten hatte ${ }^{40}$.

\section{Die "Zwischenkriegszeit"}

Während der erste Weltkrieg keinen erkennbaren Einfluss auf die Sammlungstätigkeit und das Stifterwesen hatte, bedeuteten der Beginn der Weimarer Republik und die Inflation der frühen 20er Jahre eine klare Zäsur. Sehr deutlich lässt sich dies an den Stiftungen zyprischer Antiken erkennen, die das Römisch-Germanische Zentralmuseum in Mainz bis 1918 praktisch jährlich von verschiedenen Privatpersonen, aber auch von anderen Institutionen (wie etwa dem Musée Guimet in Paris) erhielt - ohne dass Zypern je ein besonderer Schwerpunkt der Sammelaktivitäten des Museums gewesen wäre. Diese Zuwendungen brechen schlagartig ab und setzen in Mainz erst 1927/28 wieder ein. Diese Zäsur lässt sich in der Erwerbungsgeschichte anderer Museen gleichfalls finden. So erweiterten die Museen in Dresden und Kassel ihre Zypernbestände erst 1926 und das Kestner-Museum ab 1927 wieder.

Die Weltwirtschaftskrise, die Deutschland 1929/30 erreichte, markiert einen entsprechenden Einschnitt, der sich in den Zugängen der Museumssammlungen widerspiegelt. Erst 1934 kamen mit Erwerbung der insgesamt rund 1.500 Objekte umfassenden Sammlung von Carl Anton Niessen durch das Römisch-Germanische Museum in Köln wieder zyprische Antiken in eine öffentliche Sammlung. Es handelt sich dabei allerdings gerade einmal um vier kleine archaische Vasen. Auch die Zugänge der übrigen Museen, die in dieser Zeit ihre Sammlung mit zyprischen Antiken erweitern konnten, waren zumeist relativ bescheiden: 1935 kamen aus der Sammlung Friedrich Wilhelm von Bissing eine Kalksteinfigur und eine Terrakotte nach Hannover, 1936 fünf Gefäße aus Berlin durch B. Schnaps in die Antikensammlung der Universität Tübingen. Einzig die Berliner Sammlungen konnten in dieser Zeit ihre Zypernbestände namhaft erweitern, indem sie 1936/37 rund 150 Objekte, zumeist Vasen, aus der Sammlung Kouzantonos erwarben ${ }^{41}$.

\section{Zweiter Weltkrieg und Nachkriegszeit}

Auch während des zweiten Weltkriegs gelangten zyprische Antiken aus Privatbesitz in öffentliche Sammlungen. So erhielt die Universität Erlangen 1942 mehrere zyprische Vasen aus der Münchener Sammlung Dehn, die 1908 auf der Nachlassauktion der Sammlung Julius Naue erworben worden waren und wohl ursprünglich von OhnefalschRichter stammten. Noch 1944 konnte die Universität Leipzig eine spätbronzezeitliche zyprische Vase von A. Giesecke als Geschenk annehmen.

40. Etliche seiner zyprischen Antiken sind publiziert bei Nys, Recke 2010.

41. Brehme 2002, p.13. Auch das Berliner Museum für Vor- und Frühgeschichte hat zwischen 1930 und 193621 Gefäße und mehrere Fragmente von Koutsantonos (sic!) in Lefkara erworben. 
In der Nachkriegszeit wurden zyprische Antiken nur noch selten und in der Regel als Einzelstücke erworben. Eine Ausnahme bildet der Ankauf der über 100 zyprischen Objekte umfassenden Sammlung von Tischendorf im Jahre 1952 durch das KestnerMuseum; allerdings waren diese Stücke bereits seit 1914 als Dauerleihgabe in der Sammlung.

Bemerkenswert ist jedoch die Schenkung von über 30 Gefäßen an das Akademische Kunstmuseum in Bonn durch das Department of Antiquities in Nikosia. Die 1976 als „Dubletten“ ohne bekannten Fundort abgegebenen Stücke des Cyprus Museums umfassen Gefäße von der Bronzezeit bis in die byzantinische Epoche. Eine vergleichbare, um mehrere Kalksteinstatuetten, Terrakotten und Rollsiegel erweiterte Auswahl erhielt 1978 auch die Antikensammlung der Universität Gießen ${ }^{42}$. Beide Schenkungen erinnern durch ihr Bestreben, die materielle Kultur des antiken Zypern durch eine repräsentative Auswahl zu illustrieren, an die gegen Ende des 19. Jhs. von Ohnefalsch-Richter angebotenen Konvolute ${ }^{43}$.

\section{Zusammenfassung}

Betrachtet man die oben skizzierte Geschichte des Sammelns zyprischer Antiken in Deutschland, fallen mehrere Punkte ins Auge. Da ist zum einen der Umstand, dass sieht man von den wenigen Gelehrten der Jahrhundertmitte ab - die Sammeltätigkeit erst in den letzten Jahrzehnten des 19. Jhs. einsetzt und anfänglich insbesondere von den öffentlichen Museen betrieben wird, die aus einem positivistisch-wissenschaftlichen Verständnis heraus die Antike in ihrer ganzen Breite erfassen wollen. So entsteht die wissenschaftliche Beschäftigung mit den zyprischen Altertümern nicht (wie die Disziplin der Klassischen Archäologie) aus der Systematisierung bestehender, aus ästhetischen Gründen angelegter Privatsammlungen des Adels heraus, sondern ist vielmehr methodisch der sich in dieser Zeit formierenden prähistorischen Archäologie verbunden. Erst durch die von den Museen vorbereitete Popularisierung erreicht das Sammeln zyprischer Antiken auch das Bürgertum. Während gleichzeitig die Preise für qualitätvolle, figürlich bemalte griechische Keramik enorm ansteigen, sorgt die große Menge an verfügbarem Material, das vor allem durch Max Ohnefalsch-Richter auf den Markt gebracht wird, rasch für die Entstehung umfangreicher Privatsammlungen. Neben den vergleichsweise günstigen Preisen steigert der Umstand, dass die Keramik zumeist intakt oder zumindest weitgehend vollständig angeboten wird, die Attraktivität für die Käufer. Das aufgrund der Industrialisierung und des wirtschaftlichen Aufschwungs zu Wohlstand gelangte Bürgertum war Kunst und Kultur gegenüber aufgeschlossen, und

42. Nys, Recke 2010, p. 6.

43. Auch die Antikensammlung der Universität Würzburg erhielt seitens des Department of Antiquities in Nikosia Zuwendungen, doch handelte es sich hierbei lediglich um eine Sammlung von knapp 25 Scherben der Bronze- und Eisenzeit. Chronologisch noch enger ist die Schenkung von 5 neolithischen Scherben und 8 Steingeräten, die das Berliner Museum für Vor- und Frühgeschichte 1967 seitens des Department erhielt. 
so nimmt es nicht Wunder, dass in einer Zeit, in der die Gattung der Gewerbe-Museen entsteht, auch das antike Kunstgewerbe eine bedeutende Rolle für die Sammlertätigkeit spielt. Bezeichnenderweise gibt es praktisch keine adligen Sammlungen, die zyprische Antiken beinhalten ${ }^{44}$.

Mit dem vollständigen Verkauf von Ohnefalsch-Richters Sammlung 1896 an den Berliner Bankier Valentin Weisbach bricht der als nahezu unbegrenzt angesehene Nachschub des Kunsthandels ab, und die Ära der mehrere hundert Stücke umfassenden Ankäufe zyprischer Antiken ist schlagartig zu Ende. Auf den Kunstauktionen des frühen 20. Jhs. wechseln praktisch nur noch Einzelstücke den Besitzer, während größere Privatsammlungen als Dauerleihgaben oder Schenkungen an Museen gegeben oder von diesen erworben werden. Bis zum Ende des Kaiserreichs gelangen auch vermehrt kleinere Bestände zyprischer Antiken aus Privatbesitz in öffentliche Sammlungen. Inflation und Weltwirtschaftskrise unterbrechen kurzfristig diese Fluktuation, und mit den Transaktionen Mitte der dreißiger Jahre endet auch dieses Kapitel. In der Nachkriegszeit wird der zyprischen Kunst nicht mehr die hohe Wertschätzung zuteil, die sie im späten 19. und frühen 20. Jh. im Bürgertum genoss, und so verwundert es nicht, dass sich die Schenkungen des Department of Antiquities und des Cyprus Museums in Nikosia nicht an die großen öffentlichen Kunstsammlungen, sondern an die akademischen Universitätssammlungen richten. Erst allmählich wird die Zypern-Archäologie durch mehrere große Ausstellungen, die in den letzten Jahren stattgefunden haben, auch für ein breiteres Publikum wieder attraktiv gemacht.

\section{AUSSTELLUNGEN}

Die erste umfangreichere Präsentation zyprischer Antiken in Deutschland fand bereits im Rahmen der Berliner Gewerbeausstellung von 1896 statt, auf der Max OhnefalschRichter große Mengen insbesondere an Keramik ausstellte und auch verkaufte ${ }^{45}$. Obwohl dies dem von Ohnefalsch-Richter geförderten neuen Trend des Bürgertums entsprach, zyprische Antiken zu sammeln, blieb diese Ausstellung ohne Nachfolge. Erst 1938 waren anlässlich der Ausstellung "Antiken in deutschem Privatbesitz" in Berlin ${ }^{46}$ neben zahlreichen Antiken aus dem griechisch-römischen Kulturkreis auch mehrere kyprische Skulpturen und Gefäße ausgestellt ${ }^{47}$.

44. Einzig auf Schloss Fasanerie in Adolphseck befindet sich eine zyprische Amphore, die aus dem Besitz der Kaiserin Friedrich stammt. Es ist allerdings erwiesen, dass sie Kontakt zu OhnefalschRichter hatte, und es ist mehr als wahrscheinlich, dass diese Vase von ihm vermittelt wurde; vgl. Brönner 2001, p. 199. Auch die Sammlung von Herzog Alfred von Sachsen-Coburg und Gotha, zwischen 1887 und 1888 erworben, ist in unmittelbarem Zusammenhang mit den Aktivitäten Ohnefalsch-Richters zu sehen.

45. Vgl. Brönner 1999, p. 118.

46. Neugebauer 1938.

47. Nr. 1: Weibl. Kalkstein-Statuette, Slg. Freiherr von der Heydt; Nr. 2: Frauenkopf aus Kalkstein, Slg. Wilhelm Fabricius (1997, 2001 bei Christie's New York, vgl. Lafli, Recke 2005, 
Die eigenständige Thematisierung Zyperns im Rahmen einer Sonderausstellung lässt sich dann erst in einer Zeit fassen, die mit den traditionellen Wertvorstellungen bricht und auch in ihrem Kulturverständnis offen für neue Ansätze ist, die nicht das abendländische Erbe betonen. Die erste Ausstellung dieser Art fand 1968 in München statt. Unter dem Titel "Schätze aus Zypern" waren neben Exponaten aus Nikosia und verschiedenen zyprischen Provinzmuseen auch Bestände aus den beiden großen Münchener Sammlungen (Prähistorische Staatssammlung, Staatliche Antikensammlungen) und dem Römisch-Germanischen Zentralmuseum Mainz zu sehen ${ }^{48}$. Neben Antiken umfasste die Ausstellung auch Ikonen und Objekte der Volkskunst.

In leicht veränderter Form wurde die Ausstellung unter dem Titel "Schätze aus Zypern - Kunst aus acht Jahrtausenden" 1971 im Alten Museum in Ost-Berlin gezeigt ${ }^{49}$. Mit deutlich veränderter Konzeption war die Ausstellung „Schätze aus Zypern“ 1980 in Bonn zu sehen. Durch den Verzicht der Präsentation von Ikonen und volkskundlichen Objekten konzentrierte sie sich völlig auf die Antike und berücksichtigte gleichzeitig die aktuellen deutschen Forschungen auf Zypern: In zwei als "Anhänge" bezeichneten Abteilungen waren die deutschen Grabungen in Alt-Paphos und Tamassos mit über 50 Neufunden vertreten ${ }^{50}$.

In Bremen wurde unter dem Titel "Aphrodites Schwestern" 1987 eine Ausstellung mit rund 250 kyprischen Antiken gezeigt, neben Stücken aus Nikosia, Amsterdam, Kopenhagen und Paris auch Objekte aus Ost-Berlin und Karlsruhe ${ }^{51}$.

Organisiert vom Ost-Berliner Museum für Deutsche Geschichte wurde 1987/88 in verschiedenen polnischen Städten die Sonderausstellung "Zyprische Altertümer" gezeigt $^{52}$. Sie umfasste vor allem Stücke der Sammlung Weisbach / Ohnefalsch-Richter, die 1976 von Leipzig nach Ost-Berlin überführt worden waren. Der ursprüngliche Plan, die Ausstellung in weiteren Ländern des Ostblocks zu zeigen, wurde aufgrund der zeitpolitischen Entwicklungen aufgegeben, lediglich in Bad Frankenhausen (Thüringen) war sie noch zu sehen ${ }^{53}$.

Mit der Präsentation 1990 in Hannover "Die Phönizier im Zeitalter Homers" beginnen die Ausstellungen, die Zypern als Teil eines umfassenden Netzwerkes von

p. 16 Anm. 45.); Nr. 4: Bärtiger Männerkopf aus Kalkstein, Slg. Heinrich Hardt (jetzt: Museum August Kestner, Hannover); Nr. 133, 134, 136-138: Gefäße der Slg. Curt Luckow (Nr. 137 jetzt Privatbesitz Berlin, die übrigen seit 1976 Antikensammlung Dresden).

48. Spiteris 1968.

49. Ministerium für Kultur der DDR und Staatliche Museen zu Berlin (Hrsg.), Schätze aus Zypern. Kunst aus acht Jahrtausenden (Berlin 1971).

50. Grunewald 1980. - Anders als in München und Ost-Berlin wurde die Ausstellung in Bonn nicht durch eigene Exponate ergänzt.

51. Pistifidis, Ganslmayr 1987.

52. Die Ausstellung war in Warszawa / Warschau, Poznan / Posen, Wrocław / Breslau und Głogow / Glogau zu sehen; vgl. Hoffmann 2004/05, p. 337.

53. Brönner 2004/05, p. 331. 
Kultur- und Handelskontakten im östlichen Mittelmeer darstellen. In Hannover wurden hierzu etliche der bis dahin wissenschaftlich völlig unbekannten Kypriaka des KestnerMuseums gezeigt, durch Stücke aus Berlin, Brüssel, London und Paris ergänzt ${ }^{54}$. Die Ausstellung wurde 1991 auch im Hamburger Museum für Archäologie und in der Prähistorischen Staatssammlung München gezeigt. In Schloß Charlottenburg richtete die Vorderasiatische Sammlung im Museum für Vor- und Frühgeschichte Berlin 1992 eine Ausstellung mit dem Titel "Frühes Zypern zwischen Orient und Okzident" aus, bei der neben eigenen Beständen auch Objekte aus der in diesem Jahr übernommenen, umfangreichen Kollektion des ehemaligen Museums für Deutsche Geschichte (also die 1976 von Leipzig übernommene Sammlung Weisbach / Ohnefalsch-Richter) verwendet wurden ${ }^{55}$. In der Bochumer Ausstellung "Das Schiff von Uluburun” von 2005 wurden ebenfalls Fernhandelsbeziehungen im östlichen Mittelmeerraum thematisiert, wobei neben den Originalfunden der Schiffsladung als Vergleichsstücke etliche Kypriaka, insbesondere aus der Sammlung des Römisch-Germanischen Zentralmuseums in Mainz, gezeigt wurden ${ }^{56}$.

In jüngster Zeit lässt sich ein gewisses Revival der großen Zypern-Ausstellungen feststellen, die die zyprische Kunst und Kultur in der gesamten Breite darstellen. So fanden 2010 gleich zwei große Zypern-Ausstellungen statt: In Hildesheim die Ausstellung "Zypern - Insel der Aphrodite", bei der 241 Antiken $^{57}$, und in Gießen die Ausstellung "Kult-Tisch - Kyprische Keramik im Kontext", bei der 108 Antiken gezeigt wurden ${ }^{58}$. Bei letzterer wurde erstmals der Gesamtbestand einer Sammlung im Rahmen einer Sonderausstellung der Öffentlichkeit gezeigt und als Bestandskatalog publiziert. Dies ist auch bei der im Februar 2013 eröffneten Ausstellung "Von Aphrodites Insel" in Hannover der Fall, bei der die rund 200 Kypriaka des Museum August Kestner erstmals in Gänze zu sehen und im Begleitkatalog publiziert wurden ${ }^{59}$.

Obwohl es sich nicht um eine Sonderausstellung handelt, muss in diesem Zusammenhang auch die 2009 erfolgte Eröffnung der Dauerausstellung im Berliner "Neuen Museum" erwähnt werden. Hier wird auf einer Fläche von 335 Quadratmetern eine breite Auswahl aus der Zypernkollektion der Berliner Antikensammlung und des Museums für Vor- und Frühgeschichte gemeinsam präsentiert, die deutschlandweit

\section{Gehring 1990.}

55. Nagel, Strommenger 2004/05, p. 308. - Zur Ausstellung ist kein Katalog, aber eine Reihe von Führungsblättern erschienen.

56. Yalcin, Pulak, Slotta 2005.

57. Lembke 2010.

58. Nys, Recke 2010; die Ausstellung war unter dem Titel "Zypern in der Antike - Schmelztiegel der Kulturen” im September 2010 auch im Archäologischen Museum der Universität Münster zu sehen.

59. Siebert, Morstadt 2013. 
in einzigartiger Weise die kyprische Kunst und Kultur von der Bronzezeit bis zur hellenistisch-römischen Zeit in ihrer ganzen Vielfalt repräsentiert ${ }^{60}$.

\section{UNIVERSITÄRE LEHRE UND FORSCHUNGSPROJEKTE}

Das antike Zypern und seine materiellen Hinterlassenschaften bilden in Deutschland keinen zentralen Schwerpunkt in der universitären Ausbildung; entsprechende Lehrveranstaltungen werden nur sporadisch und meist in Verbindung mit Forschungsprojekten an den entsprechenden Instituten angeboten (Bochum, Erlangen, Gießen). Daher ist auch die Zahl der deutschen Dissertationen, die sich vorrangig mit einem zyprischen Thema befassen, sehr übersichtlich, wie die folgende, chronologisch geordnete Auflistung zeigt ${ }^{61}$.

- Gerhard SснміDт, Kyprische Bildwerke aus dem Heraion von Samos [Samos 7] (Diss. München 1962, publ. Bonn 1968).

- Brunhild Lewe, Studien zur archaischen kyprischen Plastik (Diss. Frankfurt/Main 1973, publ. Frankfurt 1975).

- Johann-Sebastian Kühlborn, Die kyprischen Grabreliefs (Diss. Frankfurt/Main 1974/75, unpubl.).

-Andreas PapaXenopoulos, Zypriotische Medizin in der Antike ( med. Diss. Würzburg 1982, publ. Würzburg 1981).

- Reinhard SenfF, Das Apollonheiligtum von Idalion. Architektur und Statuenausstattung eines zyprischen Heiligtums (Diss. München 1986/87, publ. Jonsered 1993).

- Melitta BröNNER, Untersuchungen zur zyprischen Kalksteinplastik von den Anfängen bis zu Beginn des 4. Jh.v. u.Z. (Diss. HU Berlin 1990, unpubl.).

- Dirk Lenz, Vogeldarstellungen in der ägäischen und zyprischen Vasenmalerei des 12.9. Jahrhunderts v. Chr. Untersuchung zu Form und Inhalt (Diss. Würzburg 1994, publ. Espelkamp 1995).

- Dimitris G. Mylonas, Archaische Kalksteinplastik Zyperns. Untersuchung zur Ikonographie, Typologie und formgeschichtlichen Entwicklung der kyprischen Rundplastik der archaischen Zeit (Diss. Mannheim 1998, Online-Publikation MATEO, Mannheim 1999).

- Dieter VIEWEger, Zur Chronologie der Nekropole von Tamassos-Lambertis, Zypern (Diss. Frankfurt/Main 1998, unpubl.).

- Katharina GiesEn, Zyprische Fibeln. Typologie und Chronologie (Diss. Tübingen 2000, publ. Jonsered 2001).

- Anja Ulbrich, Kypris. Heiligtümer und Kulte weiblicher Gottheiten auf Zypern in der kyproarchaischen und -klassischen Epoche (Königszeit) (Diss. Heidelberg 2004, publ. Münster 2008).

60. Brönner 2004/05, p. 332. Bereits 2001 (eng1.) und 2002 (dt.) ist mit Unterstützung der Leventis-Stiftung ein gemeinsamer Katalog zu den Berliner Zypernsammlungen erschienen, der ungeachtet der Besitzverhältnisse die Hauptstücke nach Gattungen gegliedert vorstellt, s. Brehme, Brönner, Karageorghis, Platz-Horster, Weisser 2001 und 2002.

61. Die Auflistung beschränkt sich auf Dissertationen, die nach dem zweiten Weltkrieg eingereicht worden sind. Wenn der Titel der Dissertationsschrift von der publizierten Fassung abweicht, wird letztere genannt. 
- Andreas Stylianou, Der Sarkophag aus Amathous als Beispiel kontaktinduzierten Wandels. Ikonographie, realienkundliche Auswertung, Stil und Typologie (Diss. Mainz 2004, publ. Mainz 2007).

- Katja Walcher, Die Architektur und Bauornamentik der Königsgräber von Tamassos auf Zypern (Diss. Erlangen 2006, publ. Rahden 2009).

- Jan-Marc Henke, Die zyprischen Terrakotten aus Milet. Neue Ansätze für die Gliederung der archaischen zyprischen Terrakotten an den ostägäischen Fundorten nach stilistischen und werkstattspezifischen Kriterien (Diss. Bochum 2009, in Druckvorbereitung).

- Annika Grewe, Sepulkrale Hofarchitekturen im Hellenismus. Eine vergleichende Untersuchung zur Architektur und Funktion ausgewählter Grabanlagen in Alexandria, Kyrene und Nea Paphos (Diss. Hamburg 2010).

- Viola Lewandowski, Die Funde aus der Nekropole von Marion (Zypern) in Berlin (Diss. FU Berlin 2012, in Druckvorbereitung).

Weitere Dissertationsvorhaben laufen an unterschiedlichen Universitäten ${ }^{62}$.

Mainz. An der Johannes-Gutenberg-Universität Mainz hat der zwischen 1997 und 2008 von der DFG geförderte Sonderforschungsbereich "Kulturelle und sprachliche Kontakte: Prozesse des Wandels in historischen Spannungsfeldern Nordostafrikas / Westasiens", insbesondere das Teilprojekt: "Das archaische Zypern im Spannungsfeld von Eigenständigkeit und Fremdimpulsen" neben zahlreichen Aufsätzen und Einzelbeiträgen auch mehrere fürZypern bedeutsame Monographien ${ }^{63}$ und Tagungsbände ${ }^{64}$ hervorgebracht.

Ebenfalls 1997 wurde das “Institut für Interdisziplinäre Zypern-Studien” in Münster eröffnet, das bereits im Mai 1996 in einer gemeinsamen Initiative der Republik Zypern und der Universität Münster gegründet worden war. Neben einem regen, nicht auf die Altertumswissenschaften beschränkten Vortragsprogramm hat das Institut mehrere Tagungen veranstaltet oder mitorganisiert und gibt eine eigene Schriftenreihe heraus ${ }^{65}$. Es beteiligt sich an dem Internationalen Forschungsprojekt "Cyprus Thesaurus", das sich der Erfassung und Dokumentation der antiken und byzantinischen Monumente Zyperns widmet. Zusammen mit Margit Krpata hat Sabine Rogge, die Geschäftsführerin des

62. Daniela Diedrich, Zyprische Hortfunde der Späten Bronzezeit. Eine Studie zu Metallhandwerk und Kult (Diss.-Projekt Erlangen-Nürnberg); Martin Eckert, Aphrodite-Heiligtümer in zypriotischen Hafenstädten: Kontaktzonen am Kreuzungspunkt der Kulturen (Diss.-Projekt Univ. Hamburg); Ivonne Stach, Kyprios charakter. Untersuchungen zur Ikonographie figürlicher Darstellungen in der cypro-archaischen Vasenmalerei (Diss.-Projekt Erlangen-Nürnberg); Beatrice Stärz, Zyprische Elfenbeinschnitzereien des 2. und frühen 1. Jahrtausends v. Chr. - Ikonographische und stilistische Untersuchungen (Diss.-Projekt Erlangen-Nürnberg).

63. Nick 2006; Stylianou, Schollmeyer 2007.

64. Höckmann, Kreikenbom 2001; Braun-Holzinger, Matthäus 2002; Bol, Kreikenbom 2004; Bol, Kleibl, Rogge 2009.

65. Die in diesem Zusammenhang wichtigsten Veranstaltungen und Publikationen: Rogge 2000; Karageorghis, Rogge 2003; Rogge 2007; Rogge 2009; Bol, Kleibl, Rogge 2009. - Eine vollständige Auflistung der Veranstaltungen und Publikationen findet sich auf der Homepage des Instituts für Interdisziplinäre Zypern-Studien, http://www.uni-muenster.de/ZypernInstitut/ 
Instituts, die Tagebücher Eugen Oberhummers von dessen beiden Zypern-Reisen aus den Jahren 1887 und 1891 ediert $^{66}$.

Hamburg. An der Universität Hamburg wurde 2003-2004 ein Forschungsprojekt mit dem Titel „Die vielgestaltige Göttin. Heiligtümer und Kulte der Aphrodite auf Zypern“ durchgeführt, bei dem geschlechterspezifische Forschungsansätze im Vordergrund standen ${ }^{67}$.

Erlangen-Nürnberg. Unter Federführung von Hartmut Matthäus fanden an der Universität Erlangen-Nürnberg seit 2004 mehrere Kongresse statt, die für die zyprische Archäologie bedeutsam und zum Teil auch bereits publiziert worden $\operatorname{sind}^{68}$, darunter jüngst auch das Nachwuchs-Kolloquium POCA $2012^{69}$. Matthäus, der durch eine Vielzahl von Publikationen einen wichtigen Beitrag zur Zypernarchäologie geliefert hat, ist mit einem Forschungsprojekt zur Königsnekropole von Tamassos und auch als Mitherausgeber an der Grabungspublikation von Tamassos beteiligt; außerdem betreut er mehrere laufende Dissertationsprojekte zu zyprischen Themen $^{70}$.

Gießen. Von der Universität Gießen aus wird die derzeit einzige deutsche Grabung auf Zypern betreut, die in Kooperation mit der VUB Brüssel in Hala Sultan Tekke stattfindet $^{71}$. Im Rahmen dieser Lehrgrabungen wurden auch mehrere BA-Arbeiten zu zyprischen Themen vergeben, die von Matthias Recke als Field Director betreut wurden. In Kooperation mit Hartmut Matthäus / Universität Erlangen-Nürnberg gibt er die Grabungspublikation von Tamassos heraus und bereitet in diesem Rahmen einen Band zu den außerstädtischen Heiligtümern vor. Eine zentrale Rolle spielen dabei die Funde aus dem Apollon-Heiligtum von Phrangissa.

Justus-Liebig-Universität Giessen

\section{Anhang: Sammlungen mit zyprischen Antiken in Deutschland}

Abkürzungen

$\begin{array}{ll}\text { CA } & \text { Cypro-Archaic } \\ \text { CC } & \text { Cypro-Classical } \\ \text { CG } & \text { Cypro-Geometric } \\ \text { CH } & \text { Cypro-Hellenistic } \\ \text { CVA } & \text { Corpus Vasorum Antiquorum } \\ \text { D } & \text { Deutschland } \\ \text { Dubia } & \text { Zweifelhaftes / Fälschungen } \\ \text { EC } & \text { Early Cypriot } \\ \text { Ker } & \text { Keramik }\end{array}$

KH Kunsthandel

LC Late Cypriot

Lg Leihgabe

MC Middle Cypriot

PB Privatbesitz

SK Skulptur, Kalkstein

Slg. Sammlung

TK Terrakotta

TL Tonlampe

66. Publiziert in Bertele, Wacker 2004.

67. M. Seifert, A. Ziel in Rogge 2007, p. 9-12.

68. Karageorghis, Matthäus, Rogge 2005; Matthäus, Oettinger, Schröder 2011.

69. Postgraduate Cypriot Archaeology (POCA), Erlangen, 23.-25.11.2012.

70. s. o., p. 89.

71.s. o., p. 89. 


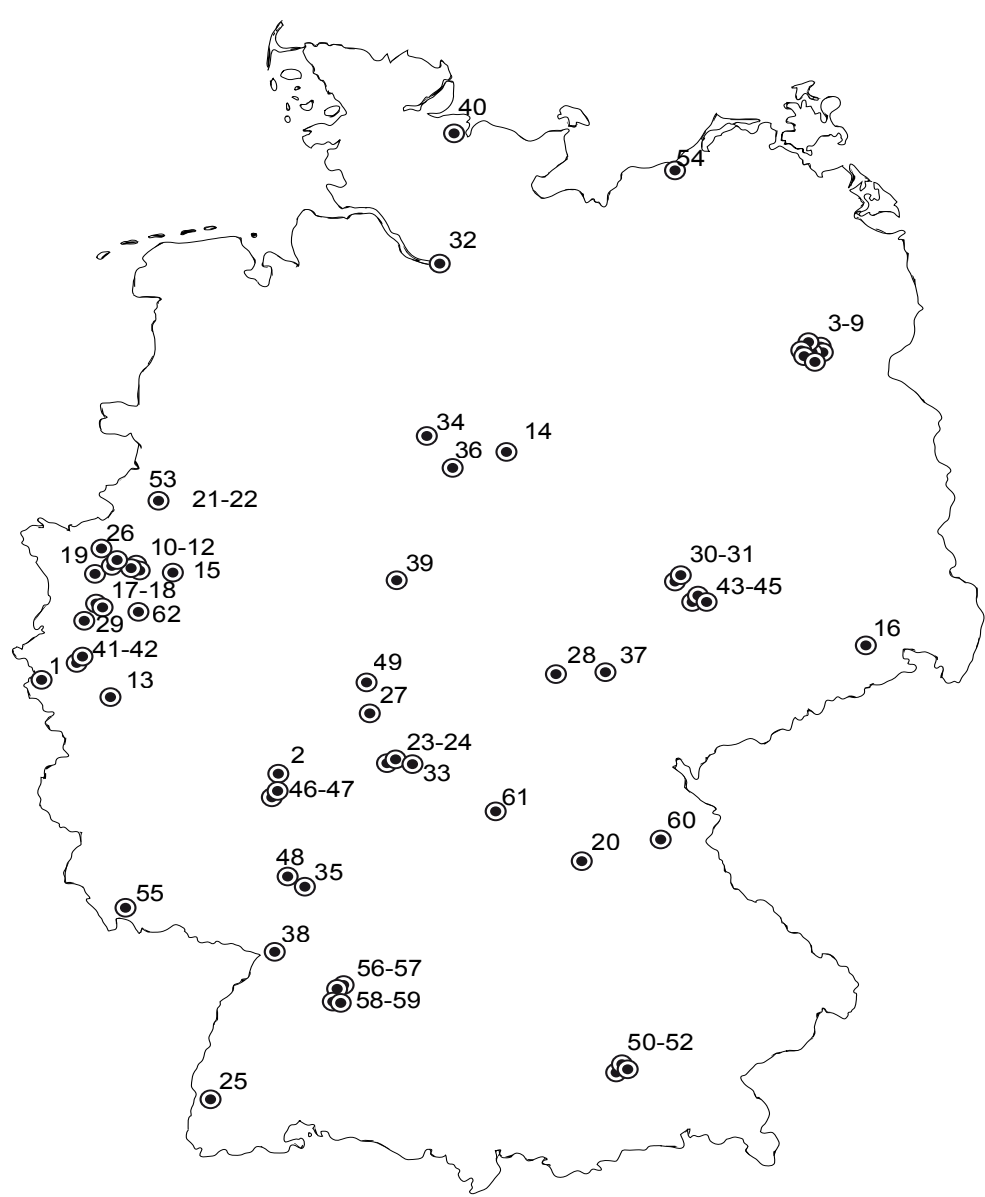

Sammlungen mit zyprischen Antiken in Deutschland.

\section{Aachen, Suermondt-Ludwig-Museum}

1. Aachen

2. Adolphseck

3-9. Berlin

10-12. Bochum

13. Bonn

14. Braunschweig

15. Dortmund

16. Dresden

17-18. Düsseldorf

19. Duisburg

20. Erlangen

21-22. Essen

23-24. Frankfurt

25. Freiburg

26. Gelsenkirchen

27. Gießen

28. Gotha

29. Grevenbroich

30-31. Halle

32. Hamburg

33. Hanau

34. Hannover

35 . Heidelberg

36. Hildesheim

37. Jena

38. Karlsruhe

39. Kassel

40. Kiel

41-42. Köln

43-45. Leipzig

46-47. Mainz

48. Mannheim

49. Marburg

50-52. München

53. Münster

54. Rostock

55. Saarbrücken

56-57. Stuttgart

58-59. Tübingen

60. Weiden

61. Würzburg

62. Wuppertal

Bestand: 1 SK (Tempelknabe), 11 Ker (MC, LC; CG; CA). Herkunft: KH Athen 1906, KH München Helbing 1910, KH 1913 Rom.

Publ.: K. Sporn (Hrsg.), Europas Spiegel. Die Antikensammlung im Suermondt-Ludwig-Museum Aachen (Wiesbaden 2005).

\section{Adolphseck, Schloß Fasanerie}

Bestand: 1 Ker (Schulterhenkelamphora WP II, CG). Herkunft: Slg. Kaiserin Friedrich, vor 1901.

Publ.: F. Brommer, CVA Schloß Fasanerie 2, D 16 (München 1959) Taf. 53,2-3.

\section{Berlin, Ägyptisches Museum}

Bestand: 1 SK (Köpfchen), 1 Silberschale, lt. Cesnola, Cypern (1879) 98 zu Tf. XIX: aus Golgoi (oder 1886 vom Antiquarium abgegeben, aus Kition).

\section{Berlin, Antikensammlung}

Bestand: Über 1200 Objekte erhalten (Kriegsverluste im 2. Weltkrieg), von EC bis zur römischen Zeit, vor allem SK, Ker, TK, aber auch Schmuck, Lampen und Bronzen, Metall, Steingeräte, Steingefäße, Spinnwirtel. Herkunft: 1845 durch Ludwig Ross (Dali, Kition); 1852 aus Slg. 
Schönborn; 1869 durch Carl Friederichs aus der Slg. L. Palma di Cesnola in Larnaka (Alambra, Idalion, Ornidia, Salamis); 1870 durch Friederichs aus Slg. Pierides / Larnaka; 1874 aus Slg. Schröder; 1878 aus Slg. H. Lang in Larnaka erworben; 1878 aus Slg. Albert Barré; 1878 aus Slg. Cesnola (aus Paphos); 1887 KH Paris (Funde der Ausgrabungen Ohnefalsch-Richters 1886 in Marion), 1887 Ankauf der Funde des Aphrodite-Heiligtums von Idalion aus der Grabung Max Ohnefalsch-Richters; 1888 Ankauf über Max Ohnefalsch-Richter der Funde eines Heiligtums bei Limniti; 1889 Grabungen im Auftrag der Berliner Museen von Ohnefalsch-Richter in Tamassos; 1884 aus Slg. Sabouroff; 1884 aus Slg. Castellani; 1893 Schenkung E. Ross; 1893 Ankauf aus Slg. Talbot Rendy; 1894 von den Berliner Museen finanzierte Grabungen in Idalion durch OhnefalschRichter; zwischen 1887 und 1890 weitere Ankäufe von Ausgrabungsfunden Ohnefalsch-Richters (Amathus, Katydhata Linou, Agios Sozomenos, Agios Nikolides, Tamassos- Agios Mnason, Tamassos-Lambertis); Funde aus Golgoi, Lapithos, Lapithos-Krini, Salamis; 1913 Schenkung aus Slg. F. L. von Gans; 1936/37 Ankauf aus Slg. Kouzantonos; 1960 KH (Goldschmuck); 1992 aus Slg. de Clercq, Paris.

Publ.: A. Furtwängler, Beschreibung der Vasensammlung im Antiquarium (Berlin 1885); Schätze aus Zypern. Kunst aus acht Jahrtausenden (Ausst.Kat. Berlin 1971); S. Brehme, M. Brönner, V. Karageorghis, G. Platz-Horster, B. Weisser, Ancient Cypriote Art in Berlin (Nicosia 2001); diess., Antike Kunst aus Zypern (Berlin 2002).

\section{Berlin, Museum für Vor- und Frïhgeschichte}

Bestand: Umfangreicher Bestand an SK, Ker (EC-CH), TK. Herkunft: Ker aus Slg. Heinrich Schliemann, Troja-Grabung; Schenkung Ferdinand Dümmler 1896 (aus Agia Paraskevi, Karpassia), Ankäufe 1930, 1931, 1936 bei Koutsantonos, Lefkara. Altbestand im 2. Weltkrieg weitgehend verloren; 1967 Schenkung des Department of Antiquities Nicosia; 1976 aus Slg. Scheye; 1992 Übernahme der Sammlung des Museums für Deutsche Geschichte, ehem. Leipzig, Völkerkunde-Museum (s. hier Nr. 45).

Publ. (s. auch hier unter Nr. 45): S. Brehme, M. Brönner, V. Karageorghis, G. Platz-Horster, B. Weisser, Ancient Cypriote Art in Berlin (Nicosia 2001); diess., Antike Kunst aus Zypern (Berlin 2002); M. Brönner, Die Sammlung zyprischer Altertümer „Ohnefalsch-Richter/Weisbach“, APA 36/37, 2004/05, 320-332; M. Brönner, Zyprische Keramikimporte in der Berliner Sammlung. in: M. Wemhoff, D. Hertel, A. Hänsel (Hrsg.) Heinrich Schliemanns Sammlung Trojanischer Altertümer - Neuvorlage Band 1 (Berlin 2008), 175-176.

\section{Berlin, Vorderasiatisches Museum}

Bestand: Basalt-Stele Sargon II aus Kition, Bronzener Fackelhalter; Herkunft: 1845 durch Ludwig Ross vermittelt.

Publ.: N. Crüsemann, Vom Zweistromland zum Kupfergraben. Jahrbuch der Berliner Museen, NF 42, 2000, Beiheft.

\section{Berlin, Münzkabinett}

Bestand: 382 Münzen, überwiegend zwischen 1840 und 1906 erworben, darunter Stücke der Sammlung Fox, Imhoof-Blumer, Löbbecke. 51 Münzen kyprischer Prägung online veröffentlicht (http://www.smb.museum/ikmk/filter_location.php?karte_id=41\&lang=de); Prägestätten sind Amathus (4), Idalion (4), Kition (6), Koinon (6), Lapethos (1), Makedonen (4), Marion (2), Nikosia (2), Paphos (2), Ptolemäer (4), Salamis (15), Soloi (1), Nachantik (5). Außerdem umfangreicher Bestand fränkischer Münzen.

Publ.: S. Brehme, M. Brönner, V. Karageorghis, G. Platz-Horster, B. Weisser, Ancient Cypriote Art in Berlin (Nicosia 2001); diess., Antike Kunst aus Zypern (Berlin 2002).

\section{Berlin, Humboldt-Universität}

Bestand: 4 Ker (EC, CA), 2 TK, (Kriegsverluste im 2. Weltkrieg). Herkunft: KH Smyrna Whitall und von Lennep, KH Athen, alle durch S. Loeschcke 1911-13.

\section{Berlin, Frei Universität}

Bestand: 1 SK (Frauenköpfchen, CC). Herkunft: Wohl durch Friedrich Wilhelm Goethert zwischen 1948 und 1974). 
Publ.: http://www.geschkult.fu-berlin.de/e/klassarch/medien/originalsammlung/ VorarbeitKatFUBerlin.pdf?1347692951

10. Bochum, Antikensammlung der Universität

Bestand: 2 Ker (CA). Herkunft: Slg. Funke 1971 / 1974.

Publ.: N. Kunisch, CVA Bochum 3, D 82 (München 2007) Taf. 1,1-4.

\section{Bochum, Münzsammlung der Universität}

Bestand: 8 Münzen, 1965 aus der Sammlung Karl Welz, Fulda (um 1920) erworben; Kleinsilber (1), Salamis (4), Kition (1), Amathus (1), Stater Nikokles (1).

\section{Bochum, Bergbaumuseum}

Bestand: Schlacken aus Apliki, Grubenholz (CC); Replik des Barren-Gotts aus Enkomi; Repliken der Uluburun-Funde.

Publ.: H.-G. Buchholz, Der Werkstoff Holz (2004) 78.

\section{Bonn, Akademisches Kunstmuseum der Universität}

Bestand: 13 SK, 93 Ker, 20 TK, Metall, 1 Münze (Stater d. Euelthon, Salamis). Herkunft: 1891/92 von Max Ohnefalsch-Richter aus dessen Grabungen, insbes. Lambertis-Nekropole Tamassos 1889), Alambra, Larnaka, Dali, Amathus; 1908 KH Athen; 1980 Schenkungen des Department of Antiquities, Nicosia.

Publ.: M. Aspris, Ein zyprischer Teller mit der Darstellung eines Webstuhls. Bonner Jahrbücher 196, 1996, 1-10.

\section{Braunschweig, Herzog Anton Ulrich-Museum}

Bestand: 1 Ker (MC). Herkunft: 1910/11 aus Slg. August Vasel (aus KH Smyrna).

Publ.: A. Greifenhagen, CVA Braunschweig, D 4 (München 1940).

\section{Dortmund, Museum für Kunst- und Kulturgeschichte}

Bestand: 1 SK, 3 Ker (CA; Dubia), 1 TL.

Publ.: K. Stähler (Hrsg.), Kunstwerke der Antike. Eine Dortmunder Sammlung (Dortmund 1988) Nr. 59-62, Nr. 114

\section{Dresden, Skulpturensammlung Albertinum}

Bestand: 1 SK, 35 Ker, 10 TK (Kriegsverluste im 2. Weltkrieg); Herkunft: durch Ohnefalsch-Richter 1891; 1976 aus Slg. Curt Luckow: vgl. K. A. Neugebauer, Antiken in deutschem Privatbesitz (Berlin 1938).

Publ.: P. Herrmann, Erwerbungen der Antikensammlungen in Deutschland, Dresden 1891, AA 1894, 23-36; K. Knoll, Die zyprischen Gefäße der Dresdner Skulpturensammlung, Jahrbuch der Staatlichen Kunstsammlungen Dresden 1996/1997, 11-29.

\section{Düsseldorf, Hetjens-Museum}

Bestand: 1 Ker (MC), aus Slg. des Kunstschriftstellers und Kanonikus Franz Bock (1823-1899).

Publ.: H. Siedentopf, CVA Nordrhein-Westfalen 1, D 49 (München 1982).

\section{Düsseldorf, Museum Kunstpalast (ehemals Gewerbemuseum)}

Bestand: 22 Goldschmuck (ursprünglich über 200 Objekte, bei einem Einbruch 1916 größtenteils gestohlen. Das Museum besitzt unveröffentlichte Schwarzweiß-Photos des kompletten Bestandes). Herkunft: Von Heinrich Frauberger auf einer Orientreise 1890 erworben, wohl über Ohnefalsch-Richter.

Publ.: H. Frauberger, Nachtrag II, in: M. Ohnefalsch-Richter, Kypros, die Bibel und Homer (Berlin 1893) 346-348, Taf. 217.

\section{Duisburg, Kultur- und Stadthistorisches Museum}

Bestand: 1 TK. Herkunft: Slg. Köhler-Osbahr.

\section{Erlangen, Antikensammlung der Universität}

Bestand: 1 SK (Lg aus PB), 5 Ker (MC, LC, CA). Herkunft: 1942 aus Slg. Dehn, davor KH München Helbing 1908: Slg. Julius Naue, wohl durch Ohnefalsch-Richter. 
Publ.: O. Dräger, CVA Erlangen 1, D 67 (München 1995); „Wechselwirkungen“ (Ausst. Erlangen 2000/2001) 17 Nr. 20 (thronender Zeus-Ammon).

21. Essen, Folkwang-Museum

Bestand: 10 Ker (MC; LC, Byz.), 1 Glas. Herkunft: Slg. Karl Ernst Osthaus, Hagen, zwischen 1902-1921; 1922 durch Folkwang-Museumsverein Essen erworben.

\section{Essen, Ruhr-Museum}

Bestand: 1 SK, 12 Ker (MC-CA; Dubia), 4 TK, 45 Metall, 1 Beingriff, 1 Steinwerkzeug. Herkunft: 1913 aus der Slg. Robert Forrer, der die Objekte Ende d. 19. Jh. über Ohnefalsch-Richter erwarb, z.T. mit Fundortangabe „Larnaka“; einige Ankäufe im KH 1961-1969, 1991; Schenkung 2001.

\section{Frankfurt, Archäologisches Museum}

Bestand: 2 SK, 17 Ker (FC-CH), 4 TL, 1 Metall, 3 Steingefäße. Herkunft: 1883 aus Slg. Milani, davor Col. Cesnola; 1892 aus Slg. Ehinger; 1907 aus Slg. Bourguignon; Slg. Stephenson; alter Bestand des Historischen Museums; 1948 aus Slg. Howell-Wills; 1968 aus KH Frankfurt (Kandelaber aus Cerveteri).

Publ.: H. Schaal, Griechische Vasen aus Frankfurter Sammlungen (Frankfurt 1923); K. Deppert, CVA Frankfurt-am Main 1, D 25 (München 1964); J. von Freeden, Antikensammlung. Ausgewählte Werke (Frankfurt 1984); S. Schäfer, Lampen der Antikensammlung (Frankfurt 1990); D. Stutzinger, Neuerwerbungen (Frankfurt 1999).

\section{Frankfurt, Liebieghaus Museum Alter Plastik}

Bestand: 2 SK, 2 Ker (MC, CA). Herkunft: 1915 aus Slg. Adolf Furtwängler; 1982 aus KH Frankfurt (bärtiger Kalksteinkopf, ,,aus Kilikien“); KH 2001.

Publ.: P. C. Bol, Bildwerke aus Stein und Stuck von archaischer Zeit bis zur Spätantike. Antike Bildwerke I (Melsungen 1983); S. Mayer-Emmerling, U. Vedder, CVA Frankfurt, Universität und Liebieghaus 4, D 66 (München 1994); R. Bol, Kopf einer Mitra-Trägerin im Liebieghaus Frankfurt, in: R. Bol u.a., Zypern, Insel im Schnittpunkt interkultureller Kontakte (Münster 2009) 13-31.

\section{Freiburg, Antikensammlung der Universität}

Bestand: 3 SK. Herkunft: Schenkung aus PB 2011.

Publ.: M. Fischer, "Archäologische Universitäts-museum und private Sammler", in F. Müller (Hrsg.), Archäologische-Universitätsmuseum und -sammlungen im Spannungsfeld von Forschung, Lehre und Offentlichkeit, Wien und Berlin, 2013, p. 555-583.

\section{Gelsenkirchen, Kunstmuseum}

Bestand: 15 Ker (EC-CC; Dubia), 1 TK, 1 TL.Als Lg in Münster, Antikensammlung der Universität. Publ.: K. Hotze, Kleinkunst der Antike (Ausst.Kat. Gelsenkirchen 1967).

\section{Gießen, Antikensammlung der Universität}

Bestand: 14 SK, 101 Ker + 75 Frgt. (EC-Byz), 6 TK, 4 TL, 8 Steingeräte, 5 Steingefäße, 2 Rollsiegel (aus Enkomi), 4 Spinnwirtel. Herkunft: 1908 KH Kassel, davor Slg. Arnold Vogell, Karlsruhe; 1909 Schenkung d. deutschen Orient-Gesellschaft (aus Gezer); 1978 Schenkung Department of Antiquities Nicosia; Lg aus PB, darunter Schenkungen d. zypr. Staatspräsidenten Erzbischofs Makarios; Schenkung 2010.

Publ.: K. Nys, M. Recke, Kult-Tisch. Kyprische Keramik im Kontext (Gießen 2010).

\section{Gotha, Schloss Friedenstein}

Bestand: 10 SK, 13 Ker (EC-CC), 3TK, Goldschmuck, 3 Gläser. Herkunft: Slg. Herzog Alfred von Sachsen-Coburg und Gotha, 1887 erworben von Ohnefalsch-Richters Grabungen in Idalion 1885, Erwerbungen 1888 auf Zypern.

Publ.: E. Rohde, CVA Gotha 1, D 24 (Berlin 1964); U. Wallenstein, Erwerbungen aus dem Mittelmeerraum, in: Ein Prinz entdeckt die Welt (Ausst.Kat. Gotha 2008).

\section{Grevenbroich, Museum}

Bestand: 6 TL. Als Lg in Münster, Antikensammlung der Universität. 


\section{Halle, Antikensammlung der Universität Robertinum}

Bestand: 1 SK, 1 Ker (CG). Herkunft: Schenkung Ludwig Ross 1851 (Kalksteinkopf aus Idalion); Lg aus PB.

\section{Halle, Münzsammlung der Universität}

Bestand: 7 Münzen Koinon (6 AE, 1 AR: Augustus-Geta).

\section{Hamburg, Museum für Kunst und Gewerbe}

Bestand: 2 SK, 28 Ker (EC-CH; Dubia), 11 TK, 1 TL, 1 Fibel, 1 Metallgefäß, 1 Glas, 2 Spinnwirtel. Herkunft: Erwerbung 1885; 1917 aus Slg. Johannes W. F. Reimers; Erwerbungen 1918, 1919, 1924, 1935, 1957, 1969; 1984 aus Slg. Walter Kropatscheck; Erwerbungen 1996, 2008, 2009.

Publ.: E. von Mercklin, Griechische und Römische Altertümer. Führer durch das Hamburgische Museum für Kunst und Gewerbe II (Hamburg 1930); W. Hornbostel (Hrsg.), Aus Gräbern und Heiligtümern (Ausst.Kat. Hamburg, Mainz 1980).

\section{Hanau, Hessisches Puppenmuseum}

Bestand: 1 SK, 5 Ker (LC, CA), 5 TK; Herkunft: KH Freiburg, G. Puhze, 1986-1995.

Publ.: M. Andres, Die Antikensammlung. Griechische, römische, altorientalische Puppen und Verwandtes (Hanau 2000) 46-56.

\section{Hannover, Museum August Kestner}

Bestand: 7 SK, 137 Ker (EC-CH, Byz), 22 TK, 4 TL, 11 Metall, 1 Elefantenstoßzahn, 1 Steingerät, 25 Glas, 2 Münzen: Kition (1), Koinon (1); Herkunft: Slg. Kestner / alter Bestand, 1900 Slg. Peter-Meyer; 1913 KH München, Helbing; 1913 KH Paris Psychas und Bear; 1914 Sammlung Tischendorf als Lg (erworben 1952); 1927 KH Berlin Lederer; 1929 KH Amsterdam, Schulmann (Tetradrachme Ptolemaios V); 1935 aus Slg. Friedrich Wilhelm von Bissing.

Publ.: U. Gehrig, H.G. Niemeyer (Hrsg.), Die Phönizier im Zeitalter Homers (Ausst.Kat. Hannover, Mainz 1990); K. Lembke (Hrsg.), Zypern, Insel der Aphrodite (Ausst.Kat. Hildesheim 2010); A.-V. Siebert, B. Morstadt (Hrsg.), Von Aphrodites Insel (Ausst.Kat. Hannover, 2013).

\section{Heidelberg, Antikenmuseum der Universität}

Bestand: 52 Ker + 7 Frgt (EC-CA), 2 TK, 3 TL, 2 Spinnwirtel. Herkunft: Zumeist vor dem 1. WK erworben in Larnaka und KH Athen, Schenkungen 1961, 1966. 2 Ker angebl. aus Kouklia / Palaepaphos, 2 Frgt. aus Enkomi.

Publ.: R. Hampe, H. Gropengießer, Aus der Sammlung des Archäologischen Instituts der Universität Heidelberg (Berlin 1967); R. Hampe und Mitarbeiter, Neuerwerbungen 1957-1970 (Mainz 1971); C. Thöne, Die griechischen und römischen Tonlampen (Mainz 2004); K. Lembke (Hrsg.), Zypern, Insel der Aphrodite (Ausst.Kat. Hildesheim 2010).

\section{Hildesheim, Roemer- und Pelizaeus-Museum}

Bestand: 7 Ker (MC-LC, CA), 2 Metall, 7 röm. Glas; Herkunft: 1898 vom Berliner Antiquarium abgetreten (2 Ker aus Hagios Nikolides und Tamassos, Lambertis), 1907 und 1913 aus Slg. Wilhelm Pelizaeus, teils in Kairo erworben, 1910 KH Kairo durch Maurice Nahmann.

Publ.: K. Lembke (Hrsg.), Zypern, Insel der Aphrodite (Ausst.Kat. Hildesheim 2010).

\section{Jena, Antikensammlung der Universität}

Bestand: 36 Ker + 44 Frgt. (EC-CA), 11 TK (EC, LC, 9 CA). Herkunft: 1908 aus KH München, Helbing (Auktion Slg. Julius Naue: 15 Ker, 1 angebl. aus Kosci nordwestlich von Larnaka); 1912 KH Paris, Psychas; 1927 aus Slg. Von Lichtenberg.

Publ.: A. Geyer (Hrsg.), Mediterrane Kunstlandschaften in der Sammlung Antiker Kleinkunst der Friedrich-Schiller-Universität (Jena 1999); V. Paul-Zinserling, Die Terrakotten der Sammlung antiker Kleinkunst (Jena 2002).

\section{Karlsruhe, Badisches Landesmuseum}

Bestand: 107 SK (CA-CH), 55 Ker (EC-CC), 56 TK (EC, MC, CA), 14 TL, 1 Bronze-Statuette, 2 Metall, 3 Steingeräte, 9 Spinnwirtel.

Herkunft: Zwischen 1853 und 1878 aus Slg. Herlan erworben (wohl aus Ägypten); 1879 durch Vermittlung A. Furtwänglers aus KH Athen; 1885 Schenkung Julius Naue (wohl von Ohnefalsch- 
Richter erworben); 1887 durch Vermittlung A. Furtwänglers Ankauf von 151 Fundstücke aus dem Aphrodite/Astarte-Heiligtum von Idalion (Grabung Max Ohnefalsch-Richters 1885); 1891 und 1895 weitere Erwerbungen bei Ohnefalsch-Richter (z.T: aus Hagia Paraskevi); 1899 aus Slg. des dt. Konsuls in Kairo, Carl August Reinhardt (angebl. aus Unterägypten); 1900 durch Vermittlung R. Hesses in Nikosia bei Martin Westorf in Nikosia erworben; 1908 aus KH München, Helbing (Auktion Slg. Julius Naue: 2 Ker); aus dem KH 1961, 1966, 1968, 1973.

Publ.: G. Hafner, CVA Karlsruhe 2, D 8 (München 1952); W. Schürmann, Katalog der kyprischen Antiken im Badischen Landesmuseum Karlsruhe. Corpus of Cypriote Antiquities 9. SIMA XX:9 (Gothenburg 1984).

\section{Kassel, Museum Schloß Wilhelmshöhe}

Bestand: 4 SK, 61 Ker (EC-CC), 11 TK, 1 Steingefäß, 2 Spinnwirtel. Herkunft: 1899 aus Nachlass Ferdinand Dümmler; 1926 durch Vermittlung Seeligs aus Nachlass Mühlmann; 1941 KH Paris; 1955 (angebl. aus Famagusta); 1984 Schenkung Oskar Liebeck; 1994 KH Zürich; 2004-2005 Lg PB.

Publ.: P. Kranz, R. Lullies, CVA Kassel 3, D 38 (München 1975); K. Yfantidis, Antike Gefäße. Eine Auswahl. Kataloge der Staatlichen Kunstsammlungen Kassel Nr. 16 (Melsungen 1990); P. Gercke, K. Yfantidis, Schenkung Dr. Oskar Liebeck Kassel 1984. Kunst in Hessen und am Mittelrhein 30, 1990, 5-18; P. Gercke, N. Zimmermann-Elseify, Antike Steinskulpturen und Neuzeitliche Nachbildungen in Kassel. Bestandskatalog (Mainz 2007).

40. Kiel, Antikensammlung der Universität

Bestand: 2 SK, 45 Ker (EC-CH) + 11 chalkolith. Frgt, 5 TK, 77 Steingeräte, 3 Spinnwirtel. Herkunft: 1896 als Lg der Berliner Museen (Polis-tis-Chrysokou; Idalion, Alambra ex Slg. Cesnola); 1906 durch F. Noack aus KH Athen; um 1935 aus Nachlass A. Bergeat († 1925); 1975 aus Nachlass Bruno Sauer († 1919); Lg aus PB.

Publ.: M. Prange, CVA Kiel 2, D 64 (München 1993).

\section{Köln, Römisch-Germanisches Museum}

Bestand: 1 Ker (CA; 3 Ker Kriegsverlust), als Lg an der Univ. Köln. Herkunft: 1934 mit insges. 15000 Antiken aus der Slg. Carl Anton Niessen erworben.

Publ.: K. Berger, F. Fless, KölnJb 28, 1995, 12-13 Nr. 3-6.

\section{Köln, Antikensammlung der Universität}

Bestand: 1 Ker (CA) als Lg des Römisch-Germanischen Museums Köln. Herkunft: 1934 aus Slg. Carl Anton Niessen.

Publ.: K. Berger, F. Fless, KölnJb 28, 1995, 12 Nr. 5.

\section{Leipzig, Antikensammlung der Universität}

Bestand: 4 SK, 18 Ker (MC-CA), Kriegsverluste 1943. Herkunft: Alter Besitz 1840/41; 1899 von Univ. Tübingen erworben; 1907 Schenkung Ed. Brockhaus, H. Credner, A. Giesecke (aus Slg. C. A. Lembessis); 1908 Schenkung Edward Perry Warren u. John Marshall (TK; Kouros-Statuette, aus Naukratis?); 1908 KH London; 1910 Schenkung Marianne Brockhaus; 1923 durch Tausch vom Völkerkunde-Museum Leipzig übernommen (ehem. Slg. Valentin Weisbach, 1896 von Max Ohnefalsch-Richter erworben); 1944 Schenkung A. Giesecke; Schenkungen 1996, 1998.

Publ.: W. Müller, CVA Leipzig 1, D 14 (Berlin 1959); E. Paul, Antike Welt in Ton. Griechische und römische Terrakotten des archäologischen Instituts in Leipzig (Leipzig o.J.).

\section{Leipzig, Grassi Museum für Angewandte Kunst}

Bestand: 2 Stier-Rhyta (LC, Base-Ring II). Herkunft: Um 1929 durch Tausch vom VölkerkundeMuseum Leipzig übernommen (davor 1896 aus Slg. Zschille).

\section{Leipzig, Museum für Völkerkunde}

Bestand: Ursprünglicher Bestand rund 1.800 Objekte (Kriegsverluste, v.a. Bronze, Blei, Glas, Gemmen und Schmuck), 1974 komplett an das Museum für Deutsche Geschichte in Ost-Berlin abgegeben, seit 1992 im Museum für Vor- und Frühgeschichte Berlin. Herkunft: 1898 als 
Schenkung von Valentin Weisbach erhalten, 1896 von Max Ohnefalsch-Richter erworben; Funde aus Kition-Kamelarga, Lapithos-Sotiros, Limniti, Mersinaki?, Nikosia-Agia Paraskevi, Pyla).

Publ.: E. Hoffmann, Zyprische Tongefäße und Terrakotten der Sammlung M. Ohnefalsch-Richter. Jb. Mus. Völkerkunde Leipzig 20, 1964, 374-412; E. Hoffmann, Zyprische Kalksteinskulpturen der Sammlung Ohnefalsch-Richter, I. Jb. Mus. Völkerkunde Leipzig 23, 1966, 113-129; diess., II. Jb. Museum Völkerkunde Leipzig 24, 1967, 187-208; diess., Zyprische Skulpturen der Sammlung Ohnefalsch-Richter aus hellenistischer Zeit. Jb. Mus. Völkerkunde Leipzig 25, 1968, 120-130.

\section{Mainz, Römisch-Germanisches Zentralmuseum}

Bestand: 195 Ker (EC-CH), 9 TK, 1 TL, 8 Goldschmuck, 21 Metall, 8 Steingeräte, 2 Steingefäße, 34 Spinnwirtel, 68 Repliken (17 Ker, 6 TK, 45 Metall), Kriegsverluste. Herkunft: 1897 Schenkung der Virchow-Stiftung, erworben von Max Ohnefalsch-Richter (aus Marion, Tamassos?); 1907 Schenkung des Musée Guimet, Paris; 1909 aus Slg. Kallenberg, 1917 aus Slg. Robert Forrer; 1939 Schenkung des Oberhessischen Museums Gießen. Eigene Anfertigung von Repliken v.a. aufgrund von Restaurierungsmaßnahmen der Werkstätten des Museums, etwa Bronzehortfund aus Enkomi, Brunnen 212 (1967) u.a. Enkomi-Funde, bronzener Dreifußkessel aus Salamis, Helm aus Königsnekropole Tamassos, Grabfund aus Psematismenos / Larnaka, ehem. Slg. Julius Naue.

Publ.: Fr. Behn, Vorhellenistische Altertümer der östlichen Mittelmeerländer (Mainz 1913); H.J. Hundt, Bericht über die Konservierung von Enkomi-Funden aus Ton und Bronze. In: C. Schaeffer, Alasia I (Paris 1971); M. Egg, Ch. Pare, Die Metallzeiten in Europa und im Vorderen Orient (Mainz 1995).

\section{Mainz, Antikensammlung der Universität}

Bestand: 1 SK, 2 Ker (CG-CA). Herkunft: Slg. Adolf Preyss, München.

Publ.: R. Hampe, E. Simon, CVA Mainz 1, D 15 (München 1959).

\section{Mannheim, Reiss-Engelhorn-Museen}

Bestand: 96 Ker (EC- 6 TK, 2 TL, 2 Goldschmuck, 12 Metall, 2 Spinnwirtel (Kriegsverluste). Herkunft: 1895 /1897 Erwerbungen von Max Ohnefalsch-Richter (Athienou, Laxia tou Riou); 1917 aus Slg. Gabriel von Max.

Publ.: K. Baumann, AA 1898, 69; A. Greifenhagen, CVA Mannheim 1, D 13 (München 1958)

\section{Marburg, Antikensammlung der Universität}

Bestand: 3 Ker (MC, CA), 2 TK. Herkunft: 1989 aus Slg. Walther Wrede (davor 1958 aus Nachlass Paul Jacobsthal, $\uparrow 1957)$

Publ.: P. Jacobsthal, AA 1923/24, 263 Abb. 2.

\section{9a. Marburg, Vorgeschichtliches Seminar}

Bestand: 10 Ker + 50 Frgt. (EC-Byz), 1 Tongerät. Herkunft: Alter Besitz (Enkomi, Famagousta, Kourion, Nea Paphos), teils aus Antikensammlung der Univ. Marburg; Schenkung aus PB.

\section{München, Staatliche Antikensammlungen}

Bestand: SK, Ker (EC-CA), TK, Kriegsverluste; Herkunft. Slg Dodwell (1 Ker); 1872 Erwerb aus Slg. Cesnola (31 Ker); 1875 Erwerb in Paris durch Hofmann (4 Ker); 1904 Schenkung v. Bissing (4 Ker aus Enkomi); 1906 Schenkung Ashmolean Museum Oxford (28 TK); 1911 Übernahme von Bayerischen Nationalmuseum (9 Ker). Slg. Paul Arndt (4 Ker); 1929 Schenkung F. Buschor (1 Ker); Slg. Pal Arndt (4 Ker); Schenkung Ludwig Curtius; alter Besitz.

Publ.: Th. Lau, Die griechischen Vasen, ihr Form- und Dekorationssystem (1877) Taf. I-II; Schätze aus Zypern. (Ausst.Kat. München 1968)

\section{München, Prähistorische Staatssammlung}

Bestand: 2 SK, 3214 Ker (EC-CA), 22 TK, 5 TL, 3 Schmuck, 23 Metall, 12 Steingeräte, 1 Steingefäß, 1 Glas, 11 Spinnwirtel. Herkunft: 1886 aus Slg. Julius Naue; um 1960 Slg. Sanzin; umfangreiche Schenkungen und Ankäufe seit 1966 (Dhenia bei Akati, Maloula, Polis). Rund 50 Gefäße als Dauer-Lg im Internationalen Keramik-Museum Weiden. 
Publ.: Schätze aus Zypern (Ausst.Kat. München 1968); G.Zahlhaas, Funde der Bronzezeit aufZypern (Ausst.Kat. München 1977); G. Zahlhaas, Keramiken der Prähistorischen Staatssammlung im Internationalen Keramik-Museum Weiden (München 1990).

\section{München, Staatliche Münzsammlung}

Bestand: 62 antike Münzen (AR aus Amathus, Kition, Paphos, Salamis; AU des Pumiathon, AU und AR Euagoras I und II; röm. Claudius I (3 AE), Vespasian 3 AE, 4 AR), Titus (2 AR), Domitian (3 AR), Trajan (4 AE), Antoninus Pius und Marc Aurel (9 AE), Iulia Domna (3 AE), Caracalla (1 AE), Geta (1 AE); auch umfangreicher Bestand an fränkischen Münzen.

\section{Münster, Antikensammlung der Universität}

Bestand: 56 Ker (EC-CC), 1 TK, 8 TL, 2 Metall, 2 Glas. Herkunft: 1965 aus Nachlass Otto Rubensohn († 1964, zw. 1901-1907 in Kairo erworben); Lg Museum Grevenbroich, Lg Stadt Gelsenkirchen.

Publ.: K. Hotze, Kleinkunst der Antike (Ausst.Kat. Gelsenkirchen 1967).

\section{Rostock, Antikensammlung der Universität, Heinrich-Schliemann-Institut}

Bestand: 1 Ker (Amphoriskos, BoR II [IV], CA). Herkunft: 1918 aus Slg. Herold (aus Unterägypten).

\section{Saarbrücken, Antikensammlung der Universität}

Bestand: 3 Ker (CA, CH), 1 Glas. Herkunft: angebl. aus Salamis.

Publ.: K. Braun, Katalog der Antikensammlung des Instituts für Klassische Archäologie der Universität des Saarlandes (1998).

\section{Stuttgart, Antikensammlung des Württembergischen Landesmuseums}

Bestand: 17 Ker (EC-CA), 3 TL. Herkunft: 1898, 1901 und 1903 als Schenkung des Geheimen Hofrats Stützel, München; 1959 aus Slg. Heinrich Scheufelen (davor 1913 KH München, Helbing, aus Rhodos).

Publ.: E. Kunze-Götte, CVA Stuttgart 1, D 26 (München 1965).

\section{Stuttgart, Münzkabinett des Württembergischen Landesmuseums}

Bestand: 4 antike Münzen (AR Amathus 5. Jh. v., AE d. Melekiathon 392-361 v. Chr., AU-Stater 4. Jh. v.; AE d. Claudius, 41-45 n. Chr.; 11 fränkische Silbermünzen der Könige Heinrich I., Heinrich II., Hugo IV., Peter I. und Jakob II. Herkunft: Erwerbungen 1949, 1988.

Publ.: http://www.landesmuseum-stuttgart.de/sammlungen/digitaler-katalog/

\section{Tübingen, Antikensammlung der Universität}

Bestand: 29 Ker, 1 TK. Herkunft: 1910 durch Paul Arndt mit Mitteln E. von Sieglins erworben; 1913 durch Karl Hähnle mit Mitteln E. v. Sieglins in Florenz erworben; 1925 aus Slg. Theodor Schreiber; 1936 durch B. Schnaps in Berlin erworben.

Publ.: K. Wallenstein, CVA Tübingen 2, D 44 (München 1979).

\section{Tübingen, Münzsammlung der Universität}

Bestand: 5 Münzen (Kition, Pumiathon; 2 AE d. Vespasian; 2 AE d. Antoninus Pius). Herkunft: Schenkung 1977 Slg. Fritz und Hildebrecht Hommel.

Publ.: D. Mannsperger, Sylloge nummorum Graecorum Tübingen 6, Phrygien-Kappadokien, römische Provinzprägungen in Kleinasien (München 1998) Nr. 4581-4585.

\section{Weiden, Internationales Keramik-Museum}

Bestand: rund 50 Ker (EC-CA), 3 TK. Herkunft: Lg der Prähistorischen Staatssammlung München. Publ.: G. Zahlhaas, Keramiken der Prähistorischen Staatssammlung im Internationalen KeramikMuseum Weiden (München 1990).

\section{Würzburg, Antikensammlung der Universität, Martin-von-Wagner-Museum}

Bestand: 17 Ker + 23 Frgt (EC-CC), 10 TK. Herkunft: aus Slg. Robert Forrer; Frgt. aus PhiliaDrakos; Schenkung des Department of Antiquities, Nicosia.

Publ.: E. Langlotz, Griechische Vasen. Martin von Wagner-Museum Universität Würzburg. (München 1932); E. Simon u. Mitarbeiter, Führer durch die Antikenabteilung des Martin von Wagner Museums der Universität Würzburg (1975); E. Schmidt, Katalog der antiken Terrakotten (Mainz 1994). 


\section{Wuppertal, Biblisch-Archäologisches Institut der Bergischen Universität}

Bestand: Studiensammlung Hans-Günter Buchholz (1919-2011), u.a. Ker, Frgt, TK, Fibeln, Steingerät; z.T. aus Nachlass Bruno Sauer († 1919), 1909 in Ägypten erworben; Repliken kyprischer Keramik im Rahmen experimenteller Archäologie.

\section{BIBLIOGRAPHIE ${ }^{72}$}

BEHN (F.), 1913, Vorhellenistische Altertümer der östlichen Mittelmeerländer. Katalog des römisch-germanischen Central-Museums 4, Mainz.

Bertele (M.), Wacker (C.), Hrsg., 2004, Die Reisetagebücher Eugen Oberhummers. Die Reisen in die Alte Welt, München.

Bol (R.), Kreikenbom (D.), Hrsg., 2004, Sepulkral- und Votivdenkmäler östlicher Mittelmeergebiete (7. Jh. v. Chr. - 1. Jh. n. Chr.). Kulturbegegnungen im Spannungsfeld von Akzeptanz und Resistenz, Mainz.

Bol (R.), Höckmann (U.), Schollmeyer (P.), Hrsg., 2008, Kult(ur)kontakte: Apollon in Milet/Didyma, Histria, Myus, Naukratis und auf Zypern, Mainz.

Bol (R.), Kleibl (K.), Rogge (S.), Hrsg., 2009, Zypern - Insel im Schnittpunkt interkultureller Kontakte. Adaption und Abgrenzung von der Spätbronzezeit bis zum 5. Jahrhundert v. Chr., Münster.

Braun-Holzinger (E. A.), Matthäus (H.), Hrsg., 2002, Die nahöstlichen Kulturen und Griechenland an der Wende vom 2. zum 1. Jahrtausend v. Chr. Kontinuität und Wandel von Strukturen und Mechanismen kultureller Interaktion, Möhnesee.

Brehme (S.), BrönNer (M.), Karageorghis (V.), Platz-Horster (G.), Weisser (B.), 2001, Ancient Cypriote Art in Berlin, Nicosia.

Brehme (S.), Brönner (M.), Karageorghis (V.), Platz-Horster (G.), Weisser (B.), 2002, Antike Kunst aus Zypern, Berlin.

BRÖNNER (M.), 1999, “Ausstellung und Verkauf zyprischer Altertümer auf der Berliner Gewerbeausstellung 1896. Die Vorgeschichte der Sammlung, OhnefalschRichter/Weisbach", Acta Praehistorica et Archaeologica 31, p. 107-123.
BrönNER (M.), 2001, “The Ohnefalsch-Richter Collection in the Museum für Vor- und Frühgeschichte, Berlin", in V. Tatton-Brown (Hrsg.), Cyprus in the 19th Century AD. Fact, Fancy and Fiction, Oxford, p. 198-206.

BRÖNNER (M.), 2004/05, “Die Sammlung zyprischer Altertümer, OhnefalschRichter/Weisbach", Acta Praehistorica et Archaeologica 36/37, p. 320-332.

Buchiolz, (H.-G.), 1989, "Max OhnefalschRichter als Archäologe auf Zypern", CCEC 11/12, p. 3-27.

Buchнolz, (H.-G.), 2000, "Max OhnefalschRichter, Altes und Neues zu seinen ersten Jahren in Zypern“, in L. Dubois, E. Masson (Hrsg.), Philokypros. Mélanges de Philologie et d'Antiquités grecques et proche-orientales dédiés à la mémoire d'Olivier Masson, Salamanca, p. 91-101.

Buchrolz (H.-G.), 2010, Die Nekropolen I, II und III. Tamassos, ein antiker Stadtstaat im Bergbaugebiet von Zypern, I, Münster.

Buchнolz (H.-G.), 2011, "Der Apollon Alasiotas von Tamassos in archaischer Zeit und Alašija im 2. vorchristlichen Jahrtausend" in Matthäus, Oettinger, Schröder 2011, p. $73-100$.

BuchHolz (H.-G.),Untied (K.), 1996, Tamassos. Ein antikes Königreich auf Zypern, Jonsered.

Cassimatis (H.), 1981, "Une tête chypriote dans une collection privée", RDAC, p. 160-168.

FitTSChen (K.), 2005, "Griechenland und der Orient - Ludwig Ross gegen Karl Otfried Müller", in H.R. Goette, O. Palagia (Hrsg.), Ludwig Ross und Griechenland, Rahden, p. 251-261.

Fivel (L.) [= Masson (O.)], 1996, "OhnefalschRichter vendeur d'antiquités chypriotes (1895)", CCEC 25, p. 29-35.

72. Die Bibliographie umfasst nicht die Literatur, die im Anhang zu den einzelnen Sammlungen aufgeführt ist. 
Franken (N.), 2011, "In neuem Licht. Zyprische Bronzen in der Antikensammlung der Staatlichen Museen zu Berlin", Archäologischer Anzeiger 2, p. 51-65.

Gehring (U.) Hrsg., 1990, Die Phönizier im Zeitalter Homers. Auss. Kestner-Museum Hannover, 14.9.-25.11.1990, Mainz.

FRÖHNER (W.), 1887, Antiquités Chypriotes. Catalogue des objets antiques trouvés à Arsinoé de Chypre, Paris.

Grunewald (C.), 1980, Schätze aus Zypern. 8 Jahrtausende, Neolithikum bis Mittelalter. Terrakotten, Vasen, Plastik, Geräte, Waffen, Schmuck. Akademisches Kunstmuseum der Universität Bonn, 5.11 -7.12.1980, Bonn.

Helbing (H), 1908, Sammlung Prof. Dr. Julius Naue. Auktion Hugo Helbing 19.5.1908, München.

Höckmann (U.), Kreikenbom (D.) Hrsg., 2001, Naukratis.Die Beziehung zu Ostgriechenland, Ägypten und Zypern in archaischer Zeit, Möhnesee.

HofFmann, (A.), 2004/05, "Die Vorgeschichtssammlung des ehemaligen Museums für Deutsche Geschichte und ihre Übernahme in das Museum für Vor- und Frühgeschichte", Acta Praehistorica et Archaeologica 36/37, p. 333-342.

KarageOrghis (V.), 1983, PalaepaphosSkales. An Iron Age Cemetery in Cyprus. Ausgrabungen in Alt-Paphos auf Zypern 3, Konstanz.

Karageorghis (V.), 1988, "Chronique des fouilles et découvertes archéologiques à Chypre en 1987", BCH 112, p. 793-855.

Karageorghis (V.), 2000, Ancient Art from Cyprus. The Cesnola Collection in The Metropolitan Museum of Art, New York.

Karageorghis (V.), Rogge (S.) Hrsg., 2003, Junge zyprische Archäologie, Münster.

Karageorghis (V.), Matthäus (H.), Rogge (S.), 2005, Cyprus: Religion and Society. From the Late Bronze Age to the End of the Archaic Period, Möhnesee.

Knoll (K.), 1996/7, "Die zyprischen Gefäße der Dresdener Skulpturensammlung“, Jahrbuch der Staatlichen Kunstsammlungen Dresden, p. 11-29.

Lafli (E.), Recke (M.), 2005, "Ein MarmorKouros in Mersin. Überlegungen zu Kilikien in spätarchaischer Zeit", Archäologischer Anzeiger 1, p. 1-24.

LembKe (K.) Hrsg., 2010, Zypern, Insel der Aphrodite, Hildesheim.

MAIER (F. G. ), 1983, "Einleitung", in T.B. Mitford, O. Masson, The Syllabic Inscriptions of Rantidi-Paphos. Alt-Paphos 2, Konstanz.

Masson (O.), 1992, "Diplomates et Amateurs d'Antiquités à Chypre vers 1866-1878", Journal des Savants, p. 123-154.

Masson (O.), 1996, "La dispersion des antiquités chypriotes : les deux collections Cesnola", CCEC 25, p. 3-27.

Masson (O.), Hermary (A.), 1988, "Le Voyage de Ludwig Ross à Chypre en 1845 et les Antiquités Chypriotes du Musée de Berlin", CCEC 9, p. 3-10.

MatthäUs (H.), 2009, "Max OhnefalschRichter und die Anfänge wissenschaftlicher Archäologie auf der Insel Zypern", in S. Rogge (Hrsg.), Zypern und der Vordere Orient im 19. Jahrhundert. Die Levante im Fokus von Politik und Wissenschaft der europäischen Staaten, Münster, p. 115-151.

MatthäUs (H.), 2011, "In Memoriam HansGünter Buchholz (1919-2011)“, CCEC 41, p. 9-16.

Matthäus (H.), Oettinger (N.), SchröDer (S.), 2011, Der Orient und die Anfänge Europas. Kulturelle Beziehungen von der Späten Bronzezeit bis zur Frühen Eisenzeit, Marburg.

Merl (A.), 2009, "Der Archäologe Ludwig Ross 1845 in Zypern auf den Spuren der Antike", in S. Rogge (Hrsg.), Zypern und der Vordere Orient im 19. Jahrhundert. Die Levante im Fokus von Politik und Wissenschaft der europäischen Staaten, Münster, p. 153-187.

MinisTerium FÜR KULTUR DER DDR UND Staatliche Museen zu Berlin (Hrsg.) 1971, Schätze aus Zypern. Kunst aus acht Jahrtausenden. Januar bis Februar 1971, Berlin. 
Myres (J.), Ohnefalsch-Richter (M.), 1899, A Catalogue of the Cyprus Museum, Oxford.

NÄF (B.), 2013, Testimonia Alt-Paphos. Ausgrabungen in Alt-Paphos 8, Mainz.

Nagel (W.), Strommenger (E.), 2005/05, “Die vorderasiatische Sammlung im Museum für Vor- und Frühgeschichte bis 1992", Acta Praehistorica et Archaeologica 36/37, p. 296-319.

Neugebauer (K. A.), 1938, Antiken in deutschem Privatbesitz. Festschrift zum 25jährigen Bestehen der Vereinigung der Freunde antiker Kunst, Berlin.

NICK (G.), 2006, Zypro-ionische Kleinplastik aus Kalkstein und Alabaster. Archäologische Studien zu Naukratis, 1, Bad Langensalza.

Nys (K.), Recke (M.), 2010, Kult-Tisch. Kyprische Keramik im Kontext, Gießen.

Nys (K.), Recke (M.), 2011, "Hala Sultan Tekke. Eine Handelsmetropole der späten Bronzezeit auf Zypern”, Spiegel der Forschung 28.2, p. 72-83.

Pistifidis (A.), Ganslmayr (H.), Hrsg., 1987, Aphrodites Schwestern und christliches Zypern. 9000 Jahre Kultur Zyperns, Bremen.

RogGe (S.), Hrsg., 2000, Zypern - Insel im Brennpunkt der Kulturen, Münster.

RoGGe (S.), 2007a, "Raubgräber oder Forscher? Archäologische Aktivitäten auf Zypern im 19. Jahrhundert", in S. Rogge (Hrsg.), Begegnungen-materielle Kulturen auf Zypern bis in die römische Zeit, Münster, p. 197-230.
RogGe (S.), Hrsg., 2007b, Begegnungen. Materielle Kultur auf Zypern bis in die römische Zeit, Münster.

RogGe (S.) Hrsg., 2009, Zypern und der Vordere Orient im 19. Jahrhundert. Die Levante im Fokus von Politik und Wissenschaft der europäischen Staaten, Münster.

RubENSOHN (O.), 1905, "Griechisch-römische Funde in Ägypten", Archäologischer Anzeiger, p. 65-70.

SChÜRMAnN (W.) 1984, Katalog der kyprischen Antiken in Badischen Landesmuseum Karlsruhe. Corpus of Cypriote Antiquities 9, Gothenburg.

Siebert (A.-V.), Morstadt (B.) Hrsg., 2013, Von Aphrodites Insel, Hannover.

SPITERIS (T.) Hrsg., 1968, Schätze aus Zypern. Ausstellung im Münchner Stadtmuseum, 1.2. - 31.3. 1968, München.

Stylianou (A.), Schollmeyer (P.), 2007, Der Sarkophag aus Amathous als Beispiel kontaktinduzierten Wandels, Der Sarkophag aus Golgoi. Zur Grabrepräsentation eines zyprischen Stadtkönigs, Mainz.

Yalcin (Ü.), Pulak (C.), Slotta (R.), Hrsg., 2005, Das Schiff von Uluburun. Welthandel vor 3000 Jahren, Bochum.

Zachariou-KaIla (E.), 2009, "Die griechischen Gelehrten und der Schutz der zyprischen Altertümer am Ende des 19. Jahrhunderts", in S. Rogge (Hrsg.), Zypern und der Vordere Orient im 19. Jahrhundert. Die Levante im Fokus von Politik und Wissenschaft der europäischen Staaten, Münster, p. 271-293. 